\title{
A Refined Model for Characterizing X-Ray Multilayers
}

\author{
Abraham L. Oren and Burton L. Henke \\ Center for X-Ray Optics \\ University of California \\ Lawrence Berkeley Laboratory \\ Berkeley, California 94720
}

\section{DISCLAIMER}

This report was prepared as an account of work sponsored by an agency of the United States Government. Neither the United States Government nor any agency thereol, nor any of their employees, makes any warranty, express or implied, or assumes any legal liability or responsibility for the accuracy, completeness, or usefulness of any information, apparatus, product, or process disclosed, or represents that its use would not infringe privately owned rights. Reierence herein to any specific commercial product, process, or service by trade name, trademark, manufacturer, or otherwise does not necessarily constitute or imply its endorsement, recom. mendation, or favoring by the United States Government or any agency thereof. The views and opinions of authors expressed herein do not necessarily state or reflect those of the United States Government or any agency thereof. 


\section{INTRODUCTION}

There has been much interest recently in the study of thin film multilayers. The interest has been spurred in part by several new endeavors for which the soft $x$-ray region of the spectrum is important. It is in this region $(\lambda=5 \AA$ to $100 \AA)$ which multilayers are useful. An example of a field in which multilayers are of increasing interest is fusion research, for much of the energy produced in fusion reactions is in the form of soft $x$-rays. Also, multilayers may be used as the optical components of the first $x$-ray laser cavity. In addition, the next generation of high-powered aynchrotron radiation sources thay use multilayers as pre-mirror power filters and monochrometor components. ${ }^{1}$

Thin film multilayem are ureful in the study of soft $x$-rays, for they can be used to select specific wavelengths of light through use of the Bragg law as discussed below. They are also useful for $x$-ray optic for they can diffract $x$-raya through large angles. They are very durable and can withotand a large $x$-rsy flux without damage.

A multilayer is an artificial crystal - a periodic sygtem of layess as depicted in Fig. 1a. In most useful multilayers the crystal is made up of layers of a low atomic numbered element (eg., C, Si) alternated with layers of a high atomic numbered element (eg., Mo, W). These are often referred to as the light and heavy sublayers, respectively, which together are referred to as bilayers. We refer to the sublayers or bilayers as simply layers whenever the meaning is clear from context. Because of the periodicity of the system, the $x$-radiation scattered from the various bilayers can constructively interfere. This occurs when the path difference of $x$-rays scattered from adjacent bilayers is an integral number of wavelengths so that the scattered rays will be in phase and their amplitudes will add. This is called the Bragg condition and can be found geometrically from Fig. Ib to be:

$$
m \lambda^{\prime}=2 d \sin \theta^{\prime}
$$

where $\lambda^{\prime}$ and $\theta^{\prime}$ are the wavelength and angle of incidence of the $x$-rays while inside the 


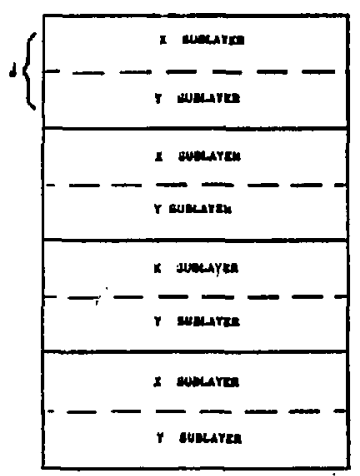

A

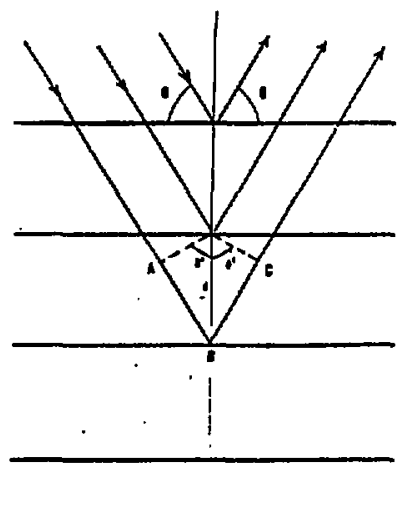

8

Figure 1. An example of a multilayer composed of four bilayers. (a) Each bilayer is made up of two sublayert of differing composition. (b) The ray reflected from layer three travals the additional distance $A B+B C$ with respect to the ray reflected from layer two.

multilayer, $d$ is the periodicity constant of the multilayer and $m$ is an integer called the order number. We find $\lambda^{\prime}$ and $\theta^{\prime}$ through application of Snell's law:

$$
n_{1} \cos \theta=n_{2} \cos \theta^{t}
$$

where $n_{1}$ is the index of refraction above the multilayer, which equals one for vacuum, and $n_{2}$ is the index of refraction in the multilayer. For soft $x$-rays, the index of refraction of materials is slightly less than one and is usually written $n_{2}=1-\delta-i \beta$, where $\delta$ and $\beta$ are on the order of $10^{-3}$. For $x$-ray refraction effects, the first order terms in $\beta$ cancel, so we may write $\cos \theta / \cos \theta^{\prime}=1-\delta=\lambda / \lambda^{\prime}$. Using the trigonometric identity $\cos \theta=\sqrt{1-\sin ^{2} \theta}$, we obtain after rearrangement:

$$
\frac{\sin \theta^{\prime}}{\lambda^{\prime}}=\frac{\sin \theta}{\lambda} \sqrt{1-\frac{2 \delta-\delta^{2}}{\sin ^{2} \theta}}
$$

The $2 d$ values of multilayers are generally in the range of 10 A to $200 \AA$, so we can achieve first order Bragg scattering $(m=1)$ through sizeable angles for all wavelengths in the soft 
x-ray region (5 Åto $100 \AA$ ).

\section{Radio-Frequency Sputtering}

Multilayers are formed most often by Radio-Frequency sputtering (R-F sputtering). In R-F sputtering, a sample of the element from which we wish to make a thin film (called the target) is bombarded with positive ions, usually $\mathrm{Ar}^{+}$. The impact of the ions frees surface atoms from the target. This process is known as sputtering. The sputtered atoms drift away from the target, colliding with and quite often sticking to whatever they come across. Thus if we place a flat substrate in the sputtering chamber at some distance in front of the target, we can build up a layer of the sputtered element upon it. Sputtered films are smooth to the order of several atomic radii, ${ }^{2}$ so they are useful for making multilayers. To make a multilayer, we alternately sputter the two elements, thus building up the periodic system of layers. The sputtering rate remain relatively stable for a given target, so we can generate the desired periodicity by just sputtering each type of layer (light or heavy) for a constant amount of time.

We now consider a more detailed description of R-F sputtering. A typical sputtering set-up is schemstically drawn in Fig. 2. The target, taxget shield and substrate upon which we wish to sputter are all placed within a vacuum chamber. The chamber is pumped down to a pressure of $10^{-7}$ Torr before sputtering to remove as much contaminating gas as powsible. After this preasure is reached, argon gas is bled into the chamber to a pressure of about $1 \mathrm{mT}$ Torr.

To begin sputtering, we merely apply a sinusoidal potential to the target with an amplitude of a few kilovolts and a frequency of about $5 \mathrm{MHz}$. If the element to be sputtered is a non-conductor, we cannot apply the potential directly to the target, so we apply it to a metal plate placed immediately behind the target as shown in Fig. 3. The target 


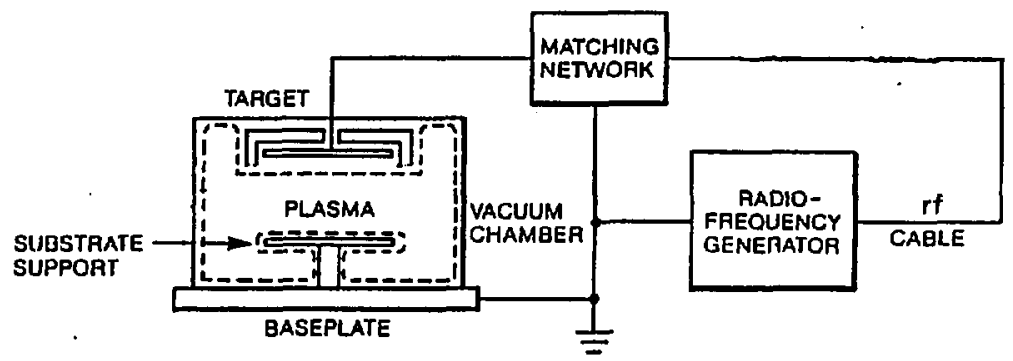

Figure 2. A typical R-F sputtering set-up.

shield, which serves to prevent sputtering from sides other than the front of the target, is grounded, as are the chamber walls and the substrate. The matching network, shown in Fig. 2, is present to equalize the load impedance with the impedance of the R-F generator to insure maximum power transfer to the target.

To understand how R-F sputtering works, we may begin by considering the part of the potential cycle where the target is at a positive voltage with respect to the chamber. If an electron is introduced inside the chamber it will be accelerated towards the target due to the field set up by the potential. The mean free path of an electron in $1 \mathrm{mT}$ Torr of argon gas is approximately five centimeters ${ }^{3}$, so the electron can gain enough energy before colliding to jonize the Argon atom it collides with. To illustrate this, consider an instant when the target potential is $+1000 \mathrm{~V}$ and let the distance between the target and the grounded baseplate of the chamber (see Fig. 2) be one meter. The potential will then 


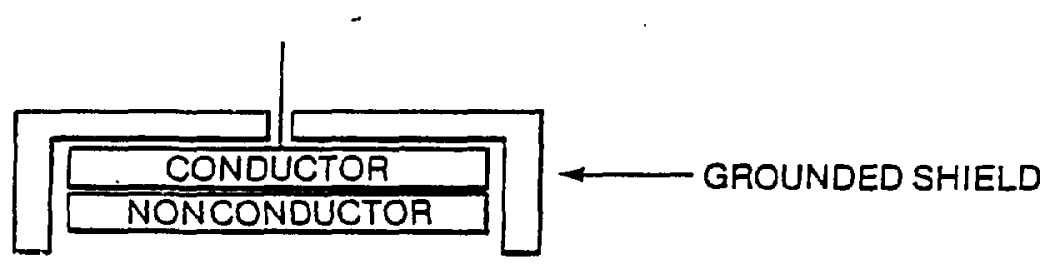

Figure 3. Target arrangement for sputtering non-conducting elements. The sputtering shield is kept at ground potential and limits sputtering to the front surface of the target.

decrease linearly to zero from the target to the baseplate with a gradient of $10 \mathrm{~V} / \mathrm{cm}$. Thus an electron accelerated for five centimeters by this field will gain $50 \mathrm{eV}$ of kinetic energy. Since the ionization energy of argon is $15.8 \mathrm{eV}$, we expect an argon atom to be ionized upon collision.

After the collision we have two electrons and an $\mathrm{Ar}^{+}$ion. The electrons will again be accelerated towards the target and can again collide with argon atoms to generate more ions and more electrons. When the sign of the potential applied to the target reverses, the electrons are accelerated away from the target and once again collide with argon atoms. We see then that a plasme is quiclily formed and maintained within the sputtering chamber. Typical sputtering plasma have one or two percent of the total number of argon atoms ionized. ${ }^{3}$

During the part of the cycle when the applied potential is negative the Ar+ ions 
will be accelerated towards the target. When they impact on the target they will often have enough energy to liberate an atom from the target surface - sputtering takes place. Howerer, the Ar+ ions quickly build up on the surface of the target, so this state does not last long. The build up of positive charge will counteract the negative potential and terminate sputtering after $\sim 10^{-7}$ seconds. ${ }^{3}$ When the potential applied to the target becomes postive, the charge build up is driven away. We can then get another $10^{-7}$ second burst of sputtering during the next cycle of the potential. Thus if the potential is cycled at a few MHz, we can get sputtering for a large fraction of the time.

The sputtering rate from a given target is relatively constant during a sputtering run. To sputter a film of a given thickness, one first determines the sputtering rate. This can be done by sputtering a thick layer onto a trial substrate. One measures the thickness of this film and finds the sputtering rate, $\tau$, by dividing this by the amount of time it was sputtered. Then one can make a film of an arbitrary thickness by merely sputtering for the appropriate amount of time. For multilayers, we divide the sputtering chamber into two regions - one with a target of the light element, the other with a target of the heavy element. We determine both rates, $\tau_{1}$ and $\tau_{2}$, then move the substrate back and forth between the regions, giving each the appropriate amount of time to obtain the desired d-spacing $(d)$ and element ratio $(r)$ :

$$
\begin{gathered}
d=\tau_{1} t_{1}+\tau_{2} t_{2} \\
r=\tau_{1} t_{1} / \tau_{2} t_{2}
\end{gathered}
$$

Several imperfections may be introduced into a multilayer structure during the manufacturing process. For instance, if the sputtering rate of either element does not stay constant while maling the multilayer, we may get a multilayer whose $d$-spacing varies from layer to layer. This can be a serious problem because now the path difference of X-rays incident upon two adjacent layers varies with depth into the crystal. The angle for 
constructive interference is thus poorly defined, so we expect the intensity of the Bragg reflection to be reduced and distorted. This effect is illustrated in Fig. 4. Fortunately this problem can be controlled by continuously monitoring the $d$-spacing during the sputtering process. 4

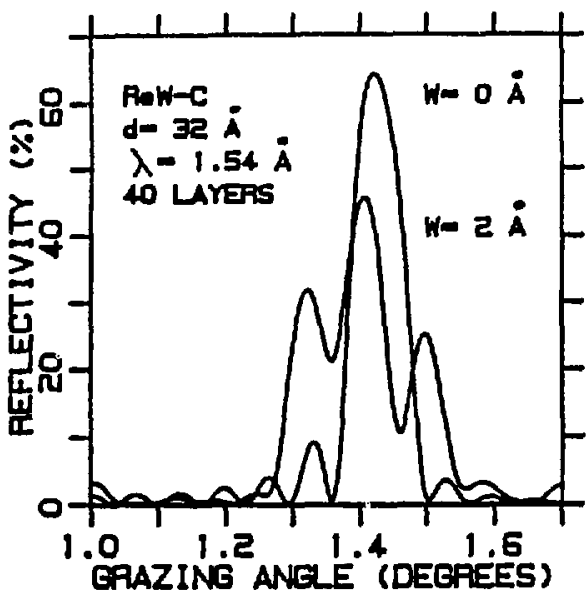

Figure 4. The effect of introducing random variations in the d-spacing on the Bragg reflection. $W$ is the Gaussian width of variations in $d$.

Other imperfections exint which cannot be so easily eliminated. When one sputters a film, its surface may not be perfectly imooth, but rather it may be marked by peals and depressions, as depicted in two dimensions in Fig. 5a. When we now sputter another element upon this surface, the new layer may fill in the depressions, as shown in Fig. 5b. The result is that we have a jagged interiace between the two layers. Even if the surface of a sputtered film is perfectly smooth, when one sputters another element onto it these new atoms may penetrate the surface and become lodged in it. The effect is that there will be a mixing of the two elements at the interiaces. Figure 6 demonstrates this sequence of 


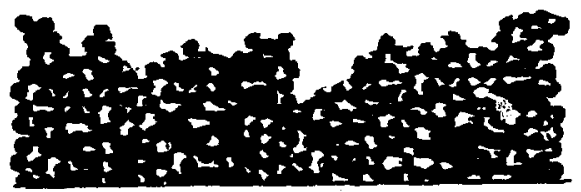

1

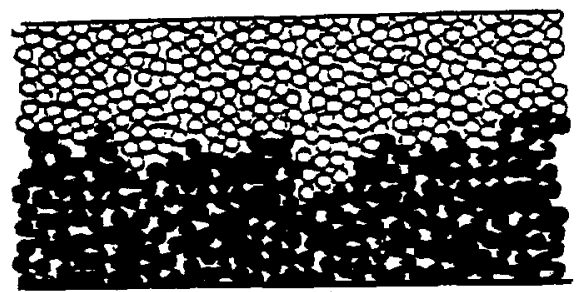

B

Figure 5. A sputtered surface may be very rough (a). When we sputter another element on to this surface (b) the depressions may be filled up, leaving a jagged interface.

events.

Either of these two effects - interface roughness or mixing - can be modelled using a transition layer between the two element layess. This is shown in. Fig. 7 where the two elements have been labelled $X$ and $Y$. Acros the transition region, the number density of the $X$ element decreases linearly from its bulk value in the pure $X$-layer to zerc at the edge of the pure Y-layer. The number density of the $\mathrm{Y}$ element correspondingly increases linearly from zero to its bulk value. We find the bulk number density, $n$, of an element from the formula:

$$
n=\frac{\rho N_{0}}{A}
$$

where $\rho$ and $A$ are the element's mass density and atomic weight respectively, and $N_{0}$ is A rogadro's number.

We can now draw a unit cell for an arbitrary multilayer as in Fig. 8. The crystal is 


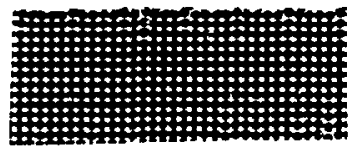

$A$
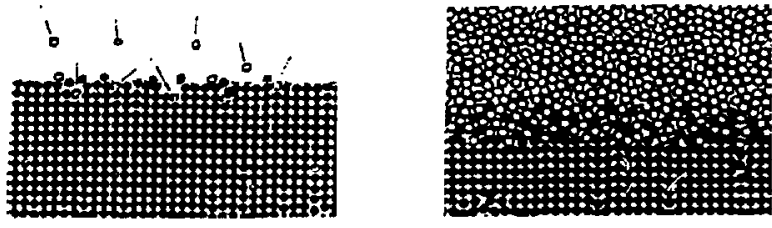

Figure 6. Atoms sputtered onto a smooth plane (a) may have enough energy to penetrate the aurface (b). This builds up a region of mixing at the interface between kwo elementi, (c).

invariant under translations in the $x$ - and $y$-directions. We may thus take the cross-section of the cell to have any arbitzisy area. The only direction of interest is the z-direction, that of depth into the multileyer. The cell is divided into four regions as shown, with transition layers interposed between the pure element layen. We define $T_{1}$ and $T_{2}$ to be the thicknesses of the two transition layers and $Y$ to be the thickness of the $Y$ element layer as indicated in Fig. 8. We can then find $X$, the thickness of the $X$ element layer from:

$$
X=d-Y-T_{1}-T_{2}
$$

where $d$ is the crystal d-spacing.

The d-spaciag of a multilayer is easily determined from the positions of the Bragg peaks by use of the Bragg condition. However the three parameters $Y, T_{1}$ and $T_{2}$ cannot be so easily determined. Changes in the values of these parameters can have dramatic 


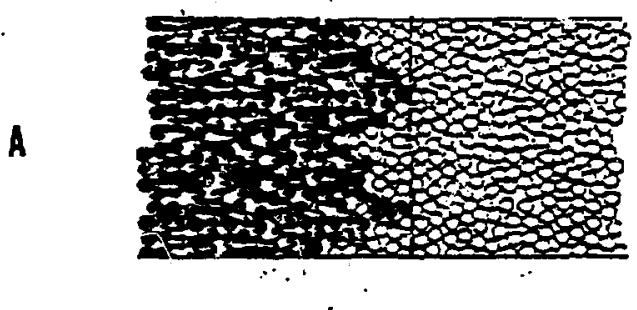

B
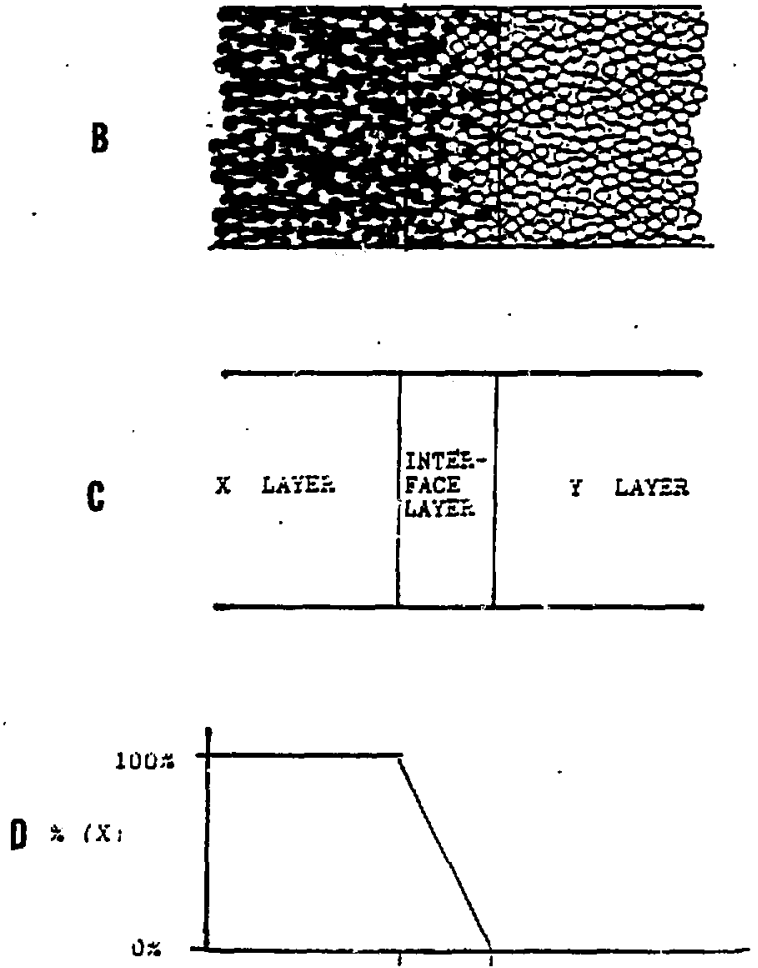

Figure 7. Either a random mixing (a) or a jagged interface (b) between elements $X$ aad $Y$ can be modelled to first order by a linear transition region interponed between the pure $X$ and pure $Y$ layess (c). The nnmber density of either element falle off across the tranaition region, as shown for $X$ in (d). Here $n_{X_{0}}$ is the bulk number density for $\mathrm{X}$. 


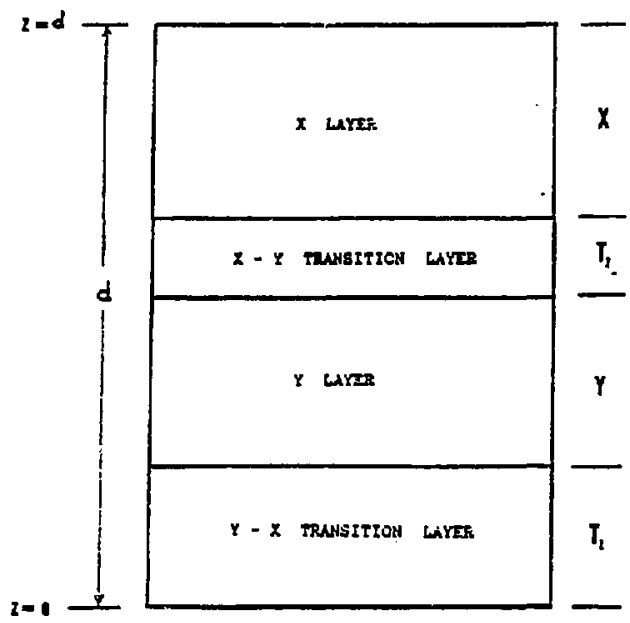

Figure 8. Unit cell for an arbitrary maltilayer composed of elements $X$ and $Y . X$ is the element at the surface of the multilayer.

effects upon the intensities of the Bragg peaks, as will be discursed later, so it is important to be able to determine them if we are to predici multilayer reflectivity. Our method is to develop a theoretical model for the reflection of $x$-rays from multilayer crystals as a function of these parameters. We then mearure the reflectivity versus angle of incidence for a given multilayer for $x$-rays of various photon energies. Next we vary the parameters of our model to best fit the theoretical reflection curres to the measured ones. We can then use this parameterizaton to predict the reflectivity of the multilayer at other photon energies. In addition, we might expect that the thicknesses of the two transition layers be approximately the same for all crystals of a given type. Thus if we characterize one multilayer, we can then generalize our parameterization to all crystals of the same type. We have the values of $T_{1}$ and $T_{2}$, so all we need to determine is the value of $Y$ to get a complete parameterization. To do this we first find the expected thickness of the Y-layer, 
$Y_{s}$, from the sputtering time and the sputtering rates

$$
Y_{S}=\pi^{t_{Y}}
$$

Some of this material will be in the Y-layer and some in the transition layers. Since the number density of the $Y$ element in the transition regions arenges $50 \%$ of that in the pure layer, we may write:

$$
Y=Y_{S}-\frac{1}{2}\left(T_{1}+T_{2}\right)
$$

The method of characterization described above bas been used effectively by Henke et. al. to model tungstea-carbon as well as other multilajer types. ${ }^{5}$ However, in their model they consider only trensition regions of equal thickress: $T_{1}=T_{2}=T$. This two parameter model works well for several crystal types, but cannot be used to parameterize molybdenum-silicon or raandium-silicon crystals. In this report we characterize these two crystal types uring the three parameter model and invertigate the important differences between them and thoee crystal types which can be fit using the two parameter model 


\section{THEORY}

Fortunately a convenient theory of I-ray difiraction from multilayer systems has been devaloped. The problem was first treated by C. G. Darwin in 1914 and then generalized to irclude tire effects of photo-abrorption by J. Prias in 1930. The resulting formula is known as the Darwin-Prins theory and can be found in standard X-Ray Physics texts ${ }^{0, T}$. This theory, with modifications made by Henke et. al," can be used for our present purposes.

\section{Scattering From A Free Electron}

We treat the problem of $x$-ray diffaction from a multilayer as a scattering problem. The diffacted intensity pattern we see will be the result of summing the amplitudes scattered from each of the atoms in the mulbilayer. The interaction between soft $x$-rays and an atom is in turn a sum of the scattering from the atom's electrons. We thus must begin by considering the scattering of $x$-rays from an electron. The dexivation that follows parillels that of Jackson?

When a plane electromagnetic wave is incident upon an electron, the electron will be accelerated along the eleetric field lines according to the Coulomb force law;

$$
F=m_{e} \mathbf{a}=e \mathbf{E}
$$

We write the electromagnetic field for the wave as:

$$
\mathrm{E}(\boldsymbol{x}, t)=\hat{\epsilon} E_{0} e^{i k \cdot x-i \omega t}
$$

where " $e$ gives the direction of polarization, $E_{0}$ is the field amplitude, $w$ is the angular frequency of the rave, and $k$ is the wave rector. Niote that ${ }^{-} \epsilon$ is perpendicular to $k$. We plug this into the force equation and solve for the acceleration:

$$
a(t)=\dot{\epsilon} \frac{e}{m} E_{0} e^{i k \cdot x-i \omega t}
$$


As expected, the electron oseillates along the polarization direction with the same frequency as the incident wave.

A charged particle under acceleration emits radiation. We can think of this radiation as the electromagnetic weve which is scattered from the electron. We find the time-averaged power scattered into solid angle d from Larmor's formula:

$$
\left\langle\frac{d P}{d \Omega}\right\rangle=\frac{\epsilon^{2}}{4 \pi c^{3}}\left\langle a^{2}\right\rangle \sin ^{2} \theta=\frac{c}{8 \pi}\left|E_{a}\right|^{2}\left(\frac{c^{2}}{m c^{2}}\right)^{2} \sin ^{2} \theta
$$

where $\theta$ is the observation angle with respect to "e, the direction of acceleration. We would like to express this result in terms of the angle of scattering, which wic will define as $2 \theta$, for reasons which will become clear later. For this purpose, we arbitrarily choose the direction of propagation for the incident meve to be along the z-aris, and let the polarization direction make an angle $\psi$ with the x-axis, as shown in Fig. 9. Froin this diagram we find:

$$
\sin ^{2} \theta=1-\sin ^{2}(2 \theta) \cos (\phi-\psi)
$$

For unpolarized incident zadiation we must arenge orer all pousible values of $\psi$, which gives us:

$$
\left\langle\frac{d P}{d \Omega}\right\rangle=\frac{c}{8 \pi}\left|E_{0}\right|^{2} r_{0}^{2} \cdot \frac{1}{2}\left(1+\cos ^{2}(2 \theta)\right)
$$

where $r_{0}=e^{2} / m c^{2}$ is the clasical electron radius and is equal to $2.813 \times 10^{-5} \mathrm{z}$. We can now obrain an expreasion for the acattered amplitude ss a function of the incident emplitude by first obtaining the time avernged Poynting vector for the scattered wave. the Poyzing rector, $S$, gives energy flow as a function of position, so we may write:

$$
\langle d P\rangle=\langle\mathbf{S}\rangle \cdot A r e a=\langle\mathbf{S}\rangle\left\langle R^{2} d \Omega\right\rangle
$$

or, rearranging,

$$
\langle\mathbf{S}\rangle=\frac{1}{R^{2}}\left\langle\frac{d P}{d \Omega}\right\rangle
$$




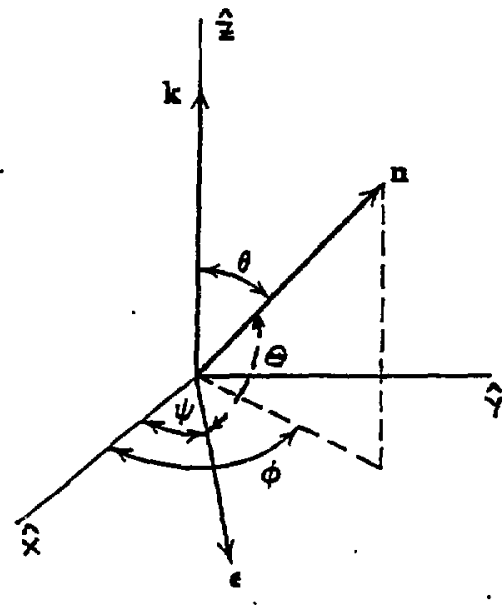

Figure 9. Geometry for the derivation of the scattering of xrays from an electron.

Here $R$ is the distnuce of the observation point from the electron. Since we can write (S) $=c|E|^{2} / 8 \pi$, we obtain an expreasion for the scattered amplitude from an electron:

$$
E=-E_{\circ} \frac{r_{0}}{R} \frac{1}{2}\left(1+\cos ^{2}(26)\right)
$$

The minus sign in this expreasion is adopted by convention. 10

\section{Scattering From An Atom}

Since only the electrons of an atom interact with soft $x$-radiation, we may think of an atom of atimic number $Z$ as a collection of $Z$ electrons. We might then expect that the amplitude scattered from an atom to be $Z$ times that scattered from an electron. However, the bound electrons of an atom car also absorb incident radiation through photo-absorption. We can model both the scattering and photo-absorption of an atom by multiplying the 
anplitude scattered from an electron by a complex scattering factor, $f=f_{1}+i f_{2}$, ${ }^{10}$ so thet:

$$
E{ }_{\text {stomie }}=-E \cdot \frac{T_{0}}{R} \frac{1}{2}\left(1+\cos ^{2} 2 \theta\right)\left(f_{1}+i f_{2}\right)
$$

The real part of the scattering factor, $f_{1}$, corresponds to scattering and the complex part, $f_{2}$, corresponds to absorption. We erpect then that $f_{1}$ will approach $z$ where the absorption $\left(f_{2}\right)$ is smoll, and to be reduced when it is large. We also expect that $f_{2}$ will suddenly inarease in value at photon energies corresponding to transitions between energy states. This is because above such an energy we can now excite another electronic transition and so more photons will be absorbed. For inatance, for energies below the $\mathrm{K}_{\mathrm{a}}$ transition of hydragen (corresponding to the excitation of the electron from the ground state to the first excited state), there can be no absorption at all, wheress above this energy there can. In this case, the $f_{2}$ value changes discontimously at the transition energy from zero to a finite value.

The $f_{1}$ and $f_{2}$ vilues for a given dement can be determined by use of Hartice - Fock calculations, 12 however these calculations are very tedious and require extensive computer resources. These values are most often found experimentally. The data from a large survey of scattering factor experiments have been compiled and averaged for elements $I$ to 94 by Fenke et. $\mathrm{al}^{10}$. We use these tabulated values of the scattering factors throughout this report. The tabulation for carbon is shown in Fig. 10 as an example of this work. Notice that the $f_{1}$ and $f_{2}$ curver behave as expected at the C-K transition (2Ti el).

\section{Scattering From A. Single Layer}

We would like now to obtain an expression for the scattering from a unit cell. We treat the unit cell as a collection of atoms, each of which scatters incident radiation, just 

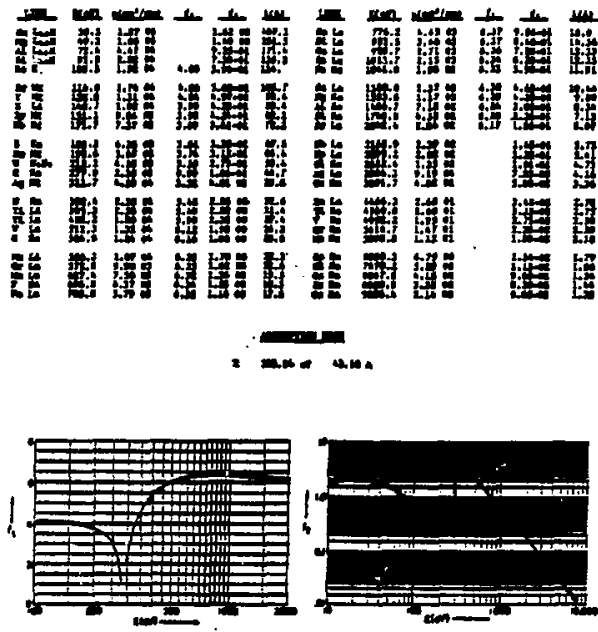

Figure 10. The atomic ecsttering factom for carbon compiled by Henke, et.al. (1982).

a we treated individual atoms as collections of electrons.

We cannot just add up the amplitude scattered from all the atoms in the unit cell because the scattered waves will have travelled different diatances and so will not be in phase. We assume the unit cell cross-section to be small compared with the wavelength of incident radiation, so that the phase difference across the unit cell laterally is small. We then only need to consider phase differences in the z-direction. For two scattering points separated by a distance $z$ along the $z$-axis and incident radiation which makes an angle $\theta$ with the $x-y$ plane, the path difference between wavefronts scattered from the two points will be $2 z \sin \theta^{\prime}$ (see Fig. 3). The phase difference is then $4 \pi z \sin \theta^{\prime} / \lambda^{\prime}$. Thus we add a phasing term to our summation:

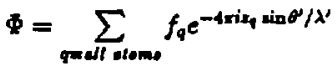

$$
\begin{aligned}
& f_{q}=f_{1 q}+i f 2 q
\end{aligned}
$$


$\Phi$ acts as an effective scattering factor for the entire wnit cell and is called the erystal structure factor. For a continuous distribution of matter, we turn the summation into an integral over the volume of the unit celli:

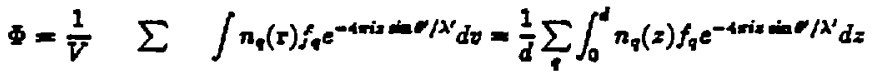

$$
\begin{aligned}
& \text { que } \\
& \text { of atom }
\end{aligned}
$$

where $n_{n}$ is the number density of the $q$ th species of atom as a function of position. We apply this expression to our perticular urit cell:

$$
\Phi=\frac{1}{d} \int_{0}^{d} n_{x}(x) f_{x} e^{-4 \operatorname{six} \sin / / x^{\prime}} d x+\frac{1}{d} \int_{0}^{d} n_{y}(x) f_{y} e^{-4 \operatorname{six} \sin \sigma / \lambda^{\prime}} d z
$$

with number densities as in Fig. 11. Performing the integration, we arrive at:

$$
\Phi=\frac{1}{d A}\left\{n_{x 0} f_{x}\left(e^{A d}-1\right)+\left(n_{y 0} f_{y}-n_{x 0} f_{x}\right)\left[\frac{1}{A T_{1}}\left(e^{A T_{1}}-1\right)-\frac{1}{A T_{2}}\left(e^{A T_{2}}-1\right) e^{A\left(Y+I_{1}\right)}\right]\right\}
$$

where $A=4 \pi i \sin \theta^{\prime} / \lambda^{\prime}$.

It is important to note that this expression was found assuming that all atoms in the unit cell have the same amplitude of radiation incident upon them - ve neglected absorption effects. Thus this expression for is only ralid in the case where the absorption per layer is small.

For our derivation of diffaction from a multilayer crystal system, we nill need $\Phi^{\prime}$, the structure factor for incident radiation from below the unit cell. For the case of negligible absorption per layer, we get the same integral as before, so $\Phi^{\prime}=\Phi$.

We can now use $\Phi$ as our "scattering factor" to determine the scattering from a single layer of unit cells. We use an ordinary Fresnel construction to add up the contributions from all the unit cells in the layer?. For an incident amplitude $T_{0}$, we get a difiracted amplitude $-i s T_{0}$ as shown in Fig. 12, where:

$$
s(\theta)=\frac{r_{0} d^{\prime} d}{\sin \theta^{\prime}} \Phi(\theta) P(2 \theta)
$$



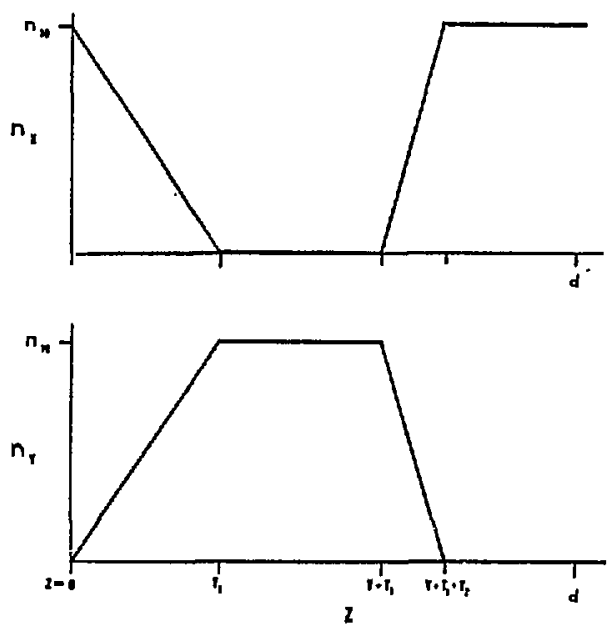

Figure 11. Number densities of the $X$ - and $Y$-elements as a function of position in the multilayer unit cell. Here $n_{20}$ and $n_{y_{0}}$ are the number densities for the bulk materials.

the $P(2 \theta)$ term accounts for the polarization of the incident radiation and is equal to 1 or $\cos (2 \theta)$ for radietion parallel or perpendicular to the scattering plane respectively. For unpolarized radiation, we average the two to get $\frac{1}{3}(1+\cos 2 \theta)$ as we had before.

The transmitted amplitude will be the sum of the incident amplitude and that of the forward scattered beam, $-i s(\theta=0) T_{0}$. We reanme $\rho(\theta=0)$ to be $\sigma$ so that the transmitted beam becomes $(1-i \sigma) T_{0}$, as shown in Fig. 12. Notice that the value of $\sigma$ is independent of the polarization of the incident beam.

\section{The Darwin-Prins Formula}

We now consider a semi-infinite system of layers, as drawn in Fig. 13. Above the $r$ th layer we have a net downward propagating wave $T_{r}$ and a net upward propagating wave 


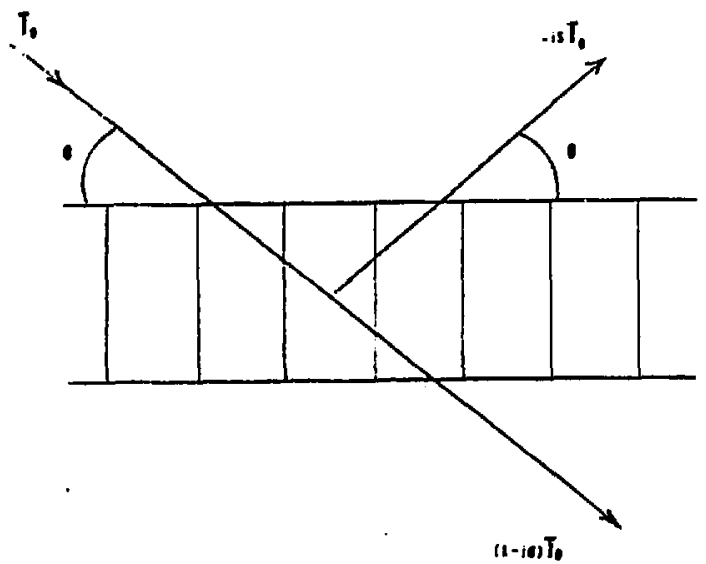

Figure 12. The absorption and reflection from a plane of unit cells.

$S_{r .}$ For the top layer, we have the incident wave $T_{0}$ and the net diffacted wave $S_{00}$ Our goal then is to solve for $S_{0}$ in terms of $T_{0}$.

Consider now the net upward propagating wave above the rth layer, $S_{r}$. Part of $S_{r}$ comes from the fraction of $T_{r}$ which is reflected from the $r$ th plane, which we have expressed as $-i s T_{r}$. Another part of $S_{r}$ comes from the fraction of $S_{r+1}$ which is transmitted upward through layer $r$. This wave has travelled the additional distance between the two layers, $d \sin \theta^{\prime}$. Thus we write for this part $(1-i \sigma) S_{r+1} \exp \left(-2 i d \sin \theta^{\prime} / \lambda^{\prime}\right)$ and the difference relation for $S_{r}$ is obtained:

$$
S_{r}=-i s T_{r}+(1-i \sigma) S_{r+1} e^{-2 \pi i d \sin / / \lambda^{\prime}}
$$

We proceed in a similar manner to obtain an expression for $T_{r}$ :

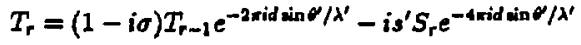

where $s^{\prime}$ corresponds to $s$, but for radiation incident from below the layer. Note that $s^{\prime}$ is 


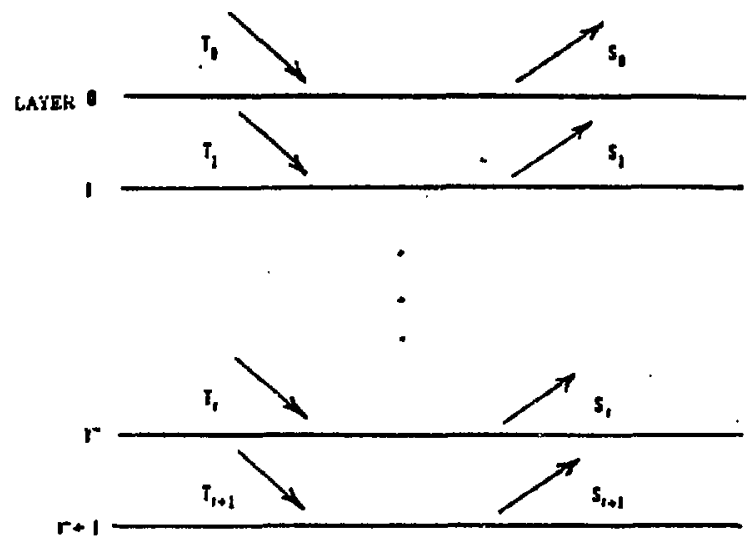

Figure 13. The amplitudes of the net upward and downward propagating waves above each layer of a multilhyer. The first layer of the multilayer is here labelled $r=0$.

identical to o because $\Phi^{\prime}$ equais $\Phi$ a we determined earlier.

We can combine these two difference expressions to get:

$$
S_{r}=-i s T_{r}+\frac{(1+i \sigma)}{-i s}\left(T_{r+1} e^{2 \pi i d \ln \psi / \lambda}-(1-i \sigma) T_{r}\right)
$$

To solve this expression with $r=0$, we need to make an assumption about the form $T_{r}$ will take. We may expect a solution where $T_{r}$ is related to $T_{0}$ by a sort of absorption factor which depends upon the number of planes traversed. This leads us to expect a solution of the form:

$$
T_{r}=T_{o} x^{r}
$$

where $x$ is the effective absorption factor per layer. We require that the real part of $x$ be less than unity, otherwise the wave will increase in amplitude as it traverses the crystal. 
Plugging in to the expression for $S$ and setting $r=0$ we get:

$$
\frac{S_{0}}{T_{0}}=\frac{-i s}{1-x(1-i \sigma) e^{-2 \pi i d \sin / / 2}}
$$

We are not yet finished, for we have no exprescion for the absorption factor, x. We procesd by assuming that any angle of interest is near the Bragg angle, designated $b_{B}$, so that:

$$
\frac{2 \pi d \sin \theta^{\prime}}{\lambda^{\prime}}=\frac{2 \pi d \sin \theta_{\theta}}{\lambda^{\prime}}+\xi=m \pi+\xi
$$

where $\xi$ is a small parameter which measures how close we are to the Bragg angle. We then see that the phase difference per layer becomes:

$$
e^{2 \pi i d d n N / \lambda^{\prime}}=e^{-i(m r+0)}=(-1)^{m} e^{-i \ell}
$$

We can naw solve the difference equations for x. We combine our second expression for $S$. with the expreasion for $T_{r}$ and the above expreasion for the phase differeace per layer, to get:

$$
(-1)^{m} e^{-i \alpha}(1-i \sigma)\left(T_{r+1}+T_{r-1}\right)=T_{r}\left\{1+e^{-2 i x}\left[(1-i \sigma)^{2}+s^{2}\right]\right\}
$$

Plugging in $T_{r}=T_{o} x^{r}$ and solving for $x$, we arrive at:

$$
\ddot{x}=(-1)^{m}\left\{1 \pm \sqrt{s^{2}-(\sigma+\xi)^{2}}\right\}
$$

where the fact that $s, \sigma$ and $\xi$ are all small quantities has been utilized. The ambiguity of the sign of the radical in this expsession is sesolved by the requirement that the real part of $x$ must be less than unity. We now plug $x$ into the expression for $S_{0} / T_{0}$, which after rearrangement appears as:

$$
\frac{S_{0}}{T_{0}}=\frac{-s}{(\sigma+\xi) \pm \sqrt{(\sigma+\xi)^{2}-s^{2}}}
$$

where

$$
\xi=\frac{2 \pi d \sin \theta^{\prime}}{\lambda^{\prime}}-m \pi
$$


This expreasion is known as the Darwin-Prins formula.

\section{Limiting The Solutlon To $N$ Layars}

The above expreasion is only valid for a cryatal system with an infinite number of layess. We would like to extend the results to finite crytal systems with $N$ layers. The argument giren below parallels that devised by Henke et. al"

We consider a semi-infinite crystal with an upper sufface at $z=N d$ and layers extending to $z=-\infty$. We aloo have an incident wave $T_{0}$ and a net diffracted wave $S_{0}$. This geometry is depicted in Fig. 14n. At the $N$ th layer of the crystal, the net downward
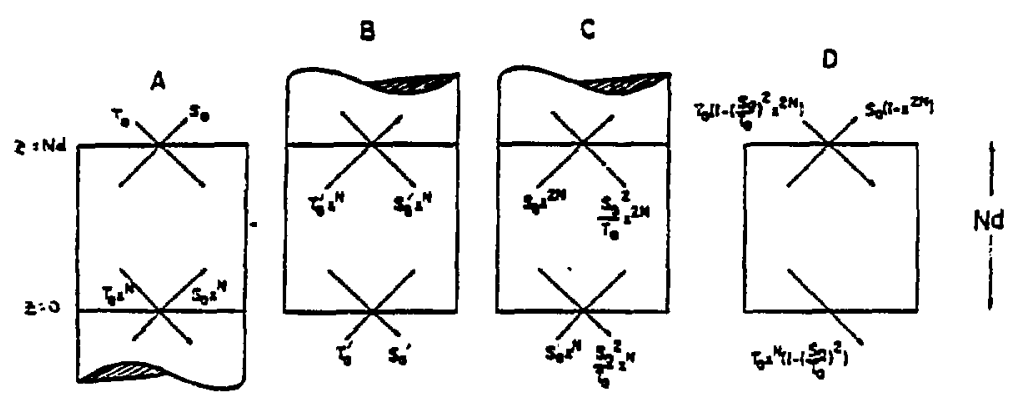

Figure 14. Geometry used to derive the Modified Darwin Prins (MDP) formula.

propagating wave will have an amplitude:

$$
T_{N}=T_{0} x^{N}
$$


where $x=(-1)^{m} 1 \pm \sqrt{s^{2}-(\sigma+\xi)^{2}}$. Below the $N$ th leyer, we again have a semi-infinite erystal just as we had at the surface of the crystal, so we expect that the ratio $S_{N} / T_{i}$ will be identical to $S_{0} / T_{0}$. Therefore the net upward propagating wave at the $N$ th layer is:

$$
S_{N}=\left(S_{0} / T_{0}\right) T_{N}=S_{0} x^{N}
$$

We now consider a semi infinite crystal vith layess extending to $z=+\infty$ as drawn in Fig. 14b. We keep the orientation of the unit cells the same as in Fig. 14a., so that now the incident beam, $T_{0}^{\prime}$, strikes the unit cells from below. The reflection from below the urit cells is the samo as for above $\left(\Phi^{\prime}=\Phi\right)$, so wo heve $S_{0}^{\prime} / T_{0}^{\prime}=S_{0} / T_{0}$, and write the waves at the Nith layer as shown in Fig. 14b. $T_{0}^{\prime}$ is arbitrary, so we choose it to have an amplitude $S_{0} x^{N}$, the amplitude of the upward propagating wave at the $N$ th layer for the crystal in Fig. 14a. the resulting set of weve amplitndes are drang in Fig. 14c.

The wave amplitudes drawn in Fig. 14 and those drawn in Fig. $14 c$ are each a ralid set of acoplituded for the $N$ layes between $z=0$ and $z=N d$. If re subtrist the amplitudes of Fig. Ite from thow of Fig. 14h, we will again bare a valid set of amplitudes for the $N$ leyers. This is what is done in Fig. 14d. Notice that we have no upward propagatiag wave at $z=0$. this is exactly the necessary boundary condition for a firite crystal of $N$ layers. Therefore, for radiation incident upon an $N$ layer crostal with amplitude $T_{0}\left(1-\left(\frac{S_{2}}{f_{0}}\right)^{2} x^{2 N}\right)$, we expect a difracted amplitude equal to $S_{0}\left(1-x^{2 N}\right)$. We take the ratio of these amplitudes to get our final result, known as the Modified Darwin-Psirs (MDP) formula:

$$
\frac{S_{o N}}{T_{0}}=\frac{\left(1-x^{2 N}\right) S_{0} / T_{0}}{1-\left(S_{0} / T_{0}\right)^{2} x^{2 N}}
$$

Once we have found the reflected amplitude, we obtain the intensity from:

$$
\frac{I}{I_{0}}=\left|\frac{S_{O N}}{T_{0}}\right|^{2}
$$




\section{The Crystal Parameter And Reflectivity}

The angular profile of a typical Bragg peal diffracted from a multilayer appears in Fig. 15. We characterize this curve with three values: its width ( $\omega)$, the peak reflectivity
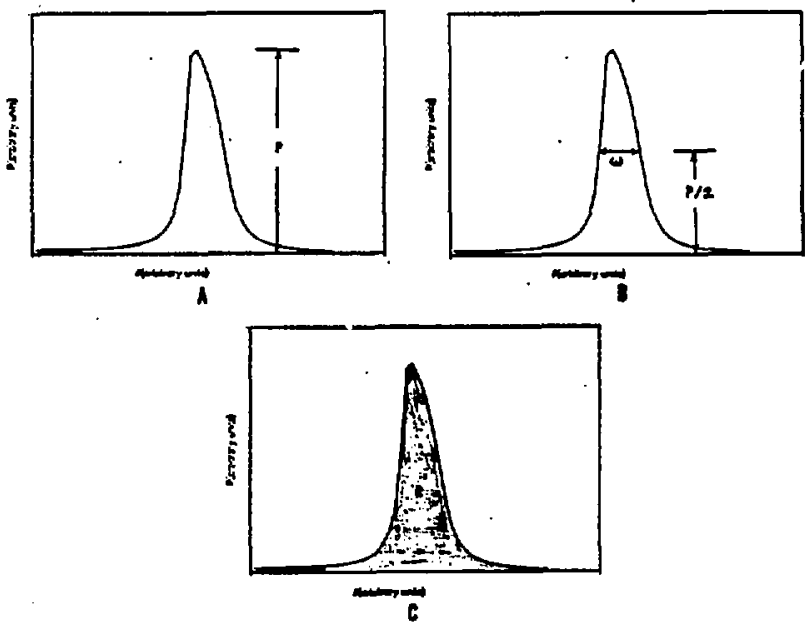

Figure 15. A typical Bragg reflection profile. The reflectivity curve is described by three values: a) the peak intensity, b) the angular width, and c) the integrated reflectivity (shaded area).

$(P)$ and the integrated reflectivity $(R)$. The width is just the full-width-at-kalf-maximum (FWHM) valus of the curve. It varies with energy and to some degree with the characteristics of the crystal and is typically on the order of 10 milliradians. The peak reflectivity is merely the maximum value of the curve and is usually expressed as percent of the incident intensity. The value of $P$ is strongly dependent upon the crystal parameters. The integrated reflectivity is the total area under the reflectivity curve, integrated over an appropriate range. Because Bragg peaks are basically Lorentzian in form, $R$ will be 
approximately related to $P$ and $\omega$ by:

$$
R=\frac{\pi}{2} P \omega
$$

We would like to understand how the reflectivity of a crystal depends upon the unit cell parameters, $Y, T_{1}$ and $T_{2}$. These parameters enter into the expression for the crystal strueture factor, $\Phi$, which is proportional to the seattering per leyer of the multilayer. We therefore begin with our previous expreasion for $\Phi$ :

$$
\left.\Phi=\frac{1}{d A}\left\{n_{s o} f_{s}\left(e^{A d}-1\right)+\left(n_{y=1} f_{y}-n_{\infty} f_{x}\right)\left[\frac{1}{A T_{1}}\left(e^{A T_{1}}-1\right)-\frac{1}{A T_{2}}\left(e^{A T_{3}}-1\right) e^{A\left(Y+T_{1}\right.}\right)\right]\right\}
$$

where

$$
A=4 \pi i \sin \theta^{\prime} / \lambda^{\prime}
$$

We begin by convidering the simpler case where $T_{1}=T_{2}=T$. With this subatitution and simple algebraic manipulations we obtain:

$$
\Phi=\frac{1}{d A}\left\{n_{x+\infty} f_{A}\left(e^{\lambda d}-1\right)+\left(n_{\infty} f_{y}-n_{\infty} f_{s}\right)\left[\frac{1}{A T}\left(e^{A T}-1\right)\left(1-e^{\mu Y+T)}\right)\right]\right\}
$$

We are interested in the reflectirity at the Brags angle. We use the Bragg condition to reespress A:

$$
\quad m \lambda^{\prime}=2 d \sin \theta^{\prime}
$$

so

$$
A=\frac{4 \pi i \sin \theta}{\lambda^{\prime}}=\frac{2 \pi i \pi}{d}
$$

The structure factor becomes:

$$
\Phi=\frac{I}{2 \pi i m}\left\{n_{s o} f_{s}\left(e^{2 \pi i m}-1\right)+\left(n_{y} f_{y}-n_{x o} f_{x}\right)\left[\frac{d}{2 \pi i m T}\left(e^{2 \pi i m T / d}-1\right)\left(1-e^{2 \pi i m(\gamma \div T) / d}\right)\right]\right\}
$$

Since $e^{2 x i m}$ is equal to one for all integers $m$, the first term in the above expression is equal to zero. We are lefi with a product of three terms:

$$
\Phi=\left(\frac{n_{x o} f_{x}-n_{y 0} f_{y}}{2 \pi m}\right)(\alpha)(\beta)
$$


where

$$
\alpha=\frac{d}{3 \pi m L}\left(e^{2 \pi i m T / d}-1\right) \text { and } \beta=1-e^{2 \pi i m(r+T) / d}
$$

The fist term tells os the scattering per layer ineseases with the contrast between scattering factor densities of the two elements which make up the multilayer. This is why multiloyers are usually made from "light" and "heavg" elements.

We would like to know for which values of the parameters $Y$ and $T$ that the amplitude of $\Phi$ equals zero. When this oceurs, the scattering per layer is zero, so there is no net scattering from the crystal and the Bragg reflection is suppressed. Because the amplitude of $\Phi$ is proportional to the amplitudes of $\alpha$ and $\beta, \Phi$ will tend towards zero whenever either of $\alpha$ or $\beta$ tends towards zero.

We will first consider $\beta$. The condition $|\beta|=0$ is expressed as:

$$
|\beta|^{2}=\left(1-e^{2 \operatorname{rim}(Y+T) / d}\right)\left(1-e^{-2 \operatorname{rin}(Y+\pi) / d}\right)=0
$$

which we solve:

$$
\begin{gathered}
2(1-\cos (2 \pi m(Y+T) / d))=0 \\
\cos (2 \pi m(Y+T) / d)=1 \\
m(Y+T) / d=\pi
\end{gathered}
$$

We see then that whenerer the suppression condition:

$$
Y+T=\frac{n d}{m}, \quad n \leq m
$$

is satisfied, $\Phi=0$, so we expect the $m$ th $B_{r a g g}$ peals to be missing. Note that $Y+T$ is merely the value of the sputtered thielness of the heary layer, $Y_{S}$ for equal interface thiclinesses:

$$
Y_{5}=Y+\frac{1}{2}\left(T_{1}+T_{2}\right)=Y+\frac{1}{2}(T+T)=Y+T
$$


We can thus easily manufacture multilayers which diffact $x$-rays, but with certain orders suppressed. For instance, to make a multilayer which will exhibit no 2nd order Bragg reflection $(m=2)$, we sputter the heavy element for an appropriate amount of time such that $Y_{S}=d / 2$. This corresponds to a $1: 1$ ratio of the sputtered thicknesses of the two elements. Figure 16 shows the peale reflectivity for the first three Bragg orders of a hypothetical Si - Mo multilayer as a function of $Y_{S} / d$. These plots show clearly that the
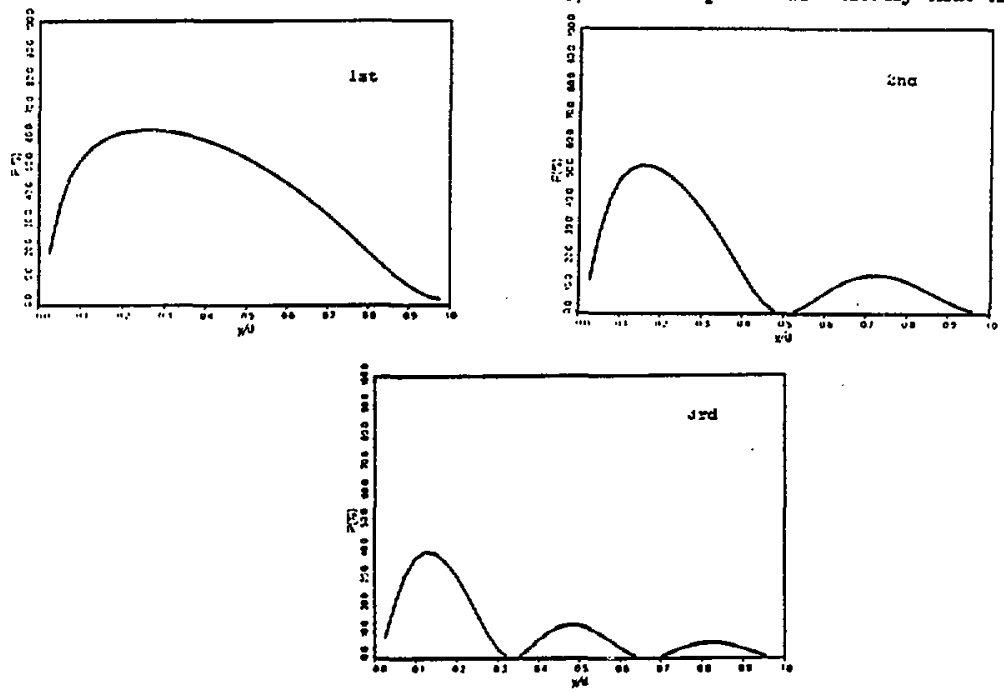

Figure 16. The dependence of the reflectivity for the first three Bragg orders on $Y_{S} / d$. Here we use 2 hypothetical Si - Mo multilayer with $d=70$ Aand $N=100$ layers.

reffectivity tends toward zero whenever $Y_{S} / d=n / m$. We also observe that the reflectivity generally decreases with increasing values of $Y_{\mathcal{S}}$. This occurs because we are increasing the amount of the heavy element, molybdenum, so the absorption per layer increases. We can quantify this by considering the forward scattering, $\Phi(\theta=0)$, which will be proportional to the absorption per layer $(\sigma)$. We write:

$$
\Phi(0)=n_{x 0} f_{x} \frac{(1-Y-T)}{d}+n_{y o} f_{Y}(Y+T) / d
$$


The value of $n_{x o} f_{z}$ will be greater than $n_{y o} f_{y}$ if the $Y$ element is the heavy element, so the abcorption increases with $Y_{s}$. With more absorption per layex, fewer layer of the crystal contribute to the Bragg peak, so it is less intense.

We can illustrate the suppression condition by considering second order reflection from a layer composed of equal heavy and light layers, each with a thickness of $d / 2$, as drawn in Fig. 17. We let ray 1 represent an incident wave scattered from the top of the

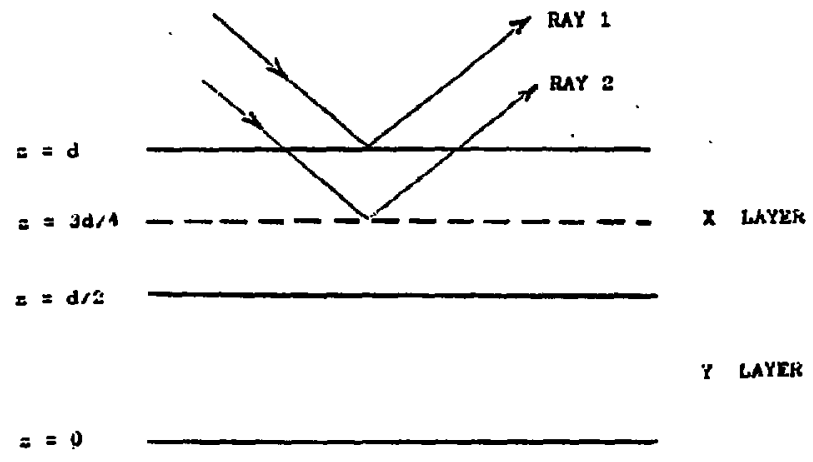

Figure 17. Destructive interference will occur between ray 1 and ray 2 at the Bragg angle for $m=2$.

light sublayer at $z=d$ and rey 2 represent an incident wave scattered from the center of this sublayer at $z=3 d / 4$. As dincussed earlier, the path difference for two waves scattered from points separated by a distance $\Delta z$ is $2 \Delta z \sin \theta^{\prime}$. In this case, $\Delta z=d / 4$ so the path difference is $d \sin \theta^{\prime} / 2$ and the phase difference is $\pi d \sin \theta^{\prime} / \lambda^{\prime}$. From the Bragg condition, $d \sin \theta^{\prime} / \lambda^{\prime}=m / 2$ which for $m=2$ equals one. Thus the phase difference is $\pi$, and we see that ray 1 and ray 2 exactly cancel each other. Now if we consider two rays scattered from 
points $x=d-\delta$ and $x=3 d / 4-\delta$ for $\delta$ between 0 and $d / 4$, we again have a separation of $\Delta x=d / 4$ so again we get cancellntion. The raye reflected from the bottom half of the sublayer cancel the rays reflected from the top half-we get pair-wise cancellation of all of the radiation reflected from the light sublayer. Since the same argument can be applied to the heavy sublayer, we get no net reflected radiation.

We now wish to examine the effects of $\alpha$, the other term in the expression for $\Phi$. We write:

$$
|\alpha|^{2}=\left(\frac{d}{2 \pi m T}\right)^{2}\left(e^{2 \pi i m T / d}-1\right)\left(e^{-2 \pi i m T / d}-1\right)
$$

which, if we let $x=2 \pi m T / d$, can be written:

$$
|\alpha|^{2}=\frac{2(1-\cos x)}{x^{2}}
$$

$|\alpha|^{2}$ is plotted in Fig. 18. It has zeroes at $z=2 n \pi$, where $n$ is a positive integer. This

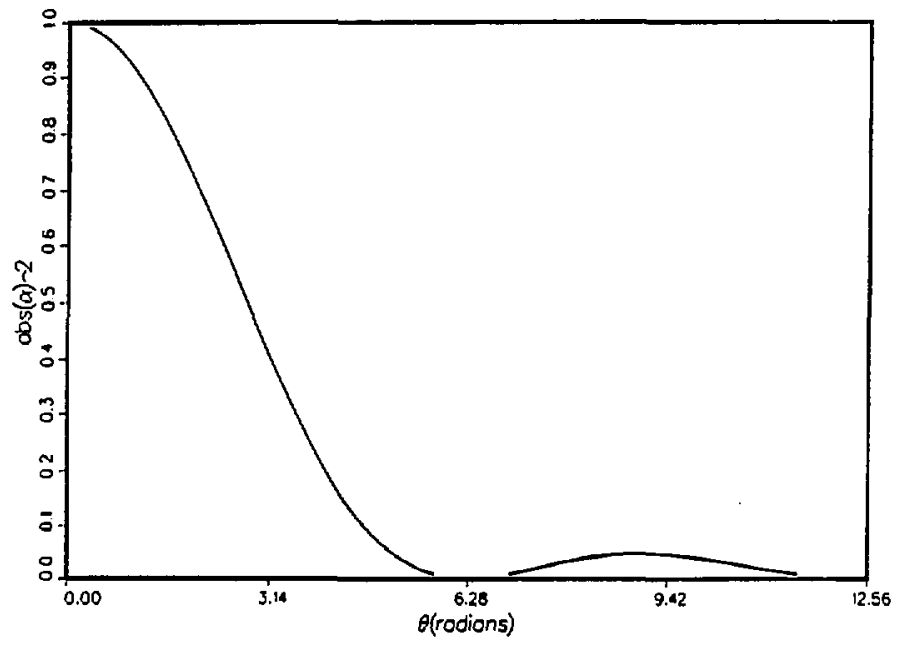

Figure 18. The function $|\alpha|^{2}$. 
leads us to our second suppression condition:

$$
T=\frac{n d}{m}, n \leq\left\lfloor\frac{m}{2}\right\rfloor
$$

If we keep $Y_{S}$ constant, then $|\Psi|^{2}$ will vary with $T$ as in Fig. 18. We can then expect the intensity of reflection from a system of layers to behave similarly. Figure 19 shows the peak reflection of the first three Bragg orders from a hypothetical multilayer as a function
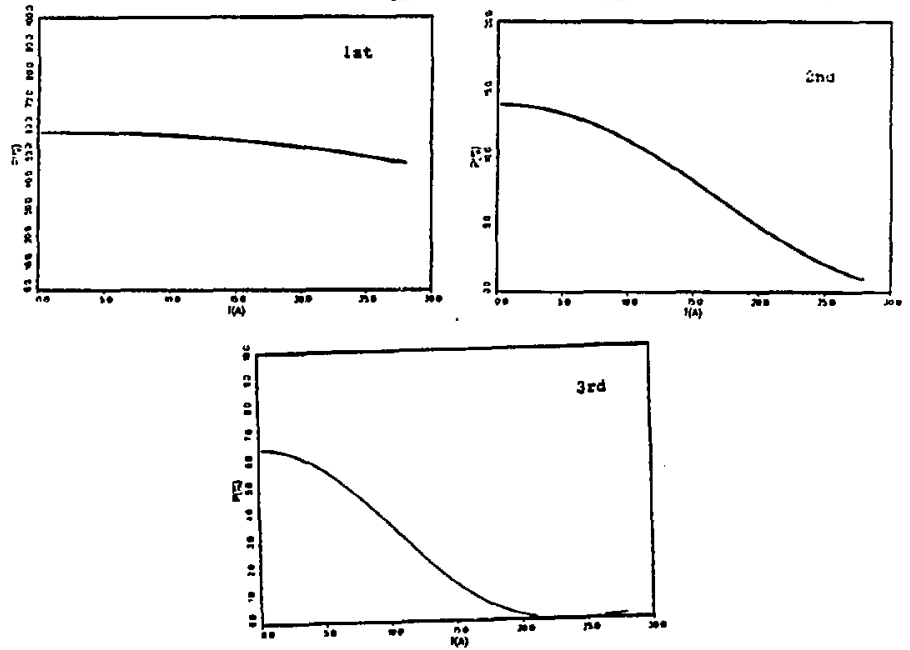

Figure 19. The dependence of the reflectivity of the first three Bragg orders on $T$. Here we use a hypothetical Si - Mo multilayer with $d=70 \AA, N=100$ layers and $Y_{S} / d=0.4$. Notice that the third order reflectivity goes to zero when $T \approx 23 \AA$. This corresponds to $T / d=1 / 3$.

of $T$ with $Y_{s} / d=0.4$. Note that practical intensities for $m$ th order reflection cannot be achieved for transition region thicknesses greater than $d / m$. We conclude that the effect of incrasing the transition layer thicknes! is basically to reduce the reflected intensity with increasing severity at higher orders.

If we now consider the unit cell where $T_{1} \neq T_{2}$, the structure factor at the $m$ th Bragg angle appears:

$$
\Phi=\frac{n_{x 0} f_{x}-n_{v o} f_{y}}{2 \pi m}\left[\frac{d}{2 \pi m T_{1}}\left(e^{2 \pi i m T_{1} / d}-1\right)-\frac{d}{2 \pi m T_{2}}\left(e^{2 \pi i m T_{2} / d}-1 j e^{2 \pi i m\left(\gamma+T_{1}\right) / d}\right]\right.
$$


We now have a difference of two terms, so the amplitude of $\Phi$ will equal zero only when both terms equal zero. Thus the addition of an asymmetry in the thiclenesses of the transition regions counteracts the effects of the previously stated suppression conditions. This is illustrated in Fig. 20. Notice that the effects of asymmetry are appreciable only at large transition layer thicknesses. Requiring that both terms in the expression for $\Phi$ go to zero gives us the suppression conditions:

$$
r_{1}=\frac{l d}{m} \text { and } T_{2}=\frac{\mathrm{md}}{\mathrm{m}}
$$

where $l$ and $n$ are integers, and $l+n \leq m$.
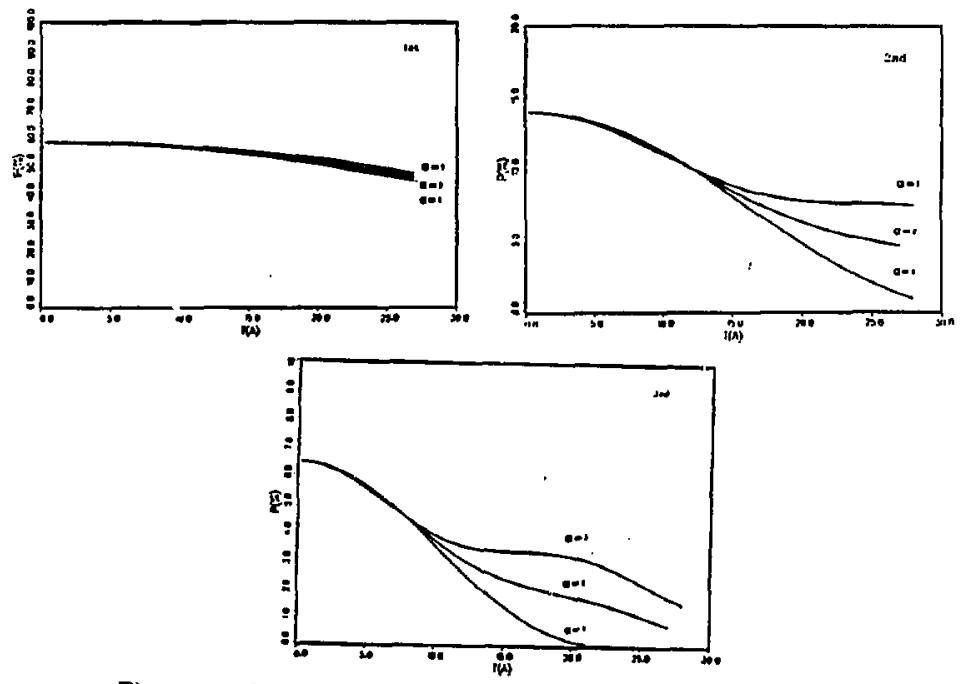

Figure 20. The reflectivity of the multilayer in Fig. 19 as a function of $T_{a y}=\frac{1}{2}\left(T_{1}+T_{2}\right)$, for various ratios of the two transition regions. Here we define the asymmetry parameter, $\alpha$, to be $T_{1} / T_{2}$.

We summarize the relationship between the crystal parameters and reflectivity from a multilayer as follows:

1. When $T_{1}=T_{2}=T$, the $m$ th order is suppressed when $Y_{S}=n d / m$. 
2. Reflectivity generally decreases as the amount of the hesvy clement increases.

3. Reflectivity decreasen as $T$ incresses. This effect is increasingly severe at bigher orders.

4. When $T_{1} \neq T_{2}$, the effecis of 1)-and 3) are decreased. 
We determine the crystal parameters, $Y, T_{1}$, and $T_{2}$, for a given multilayer by actually measuring its reflectivity at several photon energies and then varying the parameters in the MDP theory to best fit the predicted reflectivity to the measured values. In this section we discuss the experimental methods which are used to obtain multilayer reflectivity values.

We use an $x$-ray spectrograph, drawn schematically in Fig. 21, to obtain $x$-ray re-

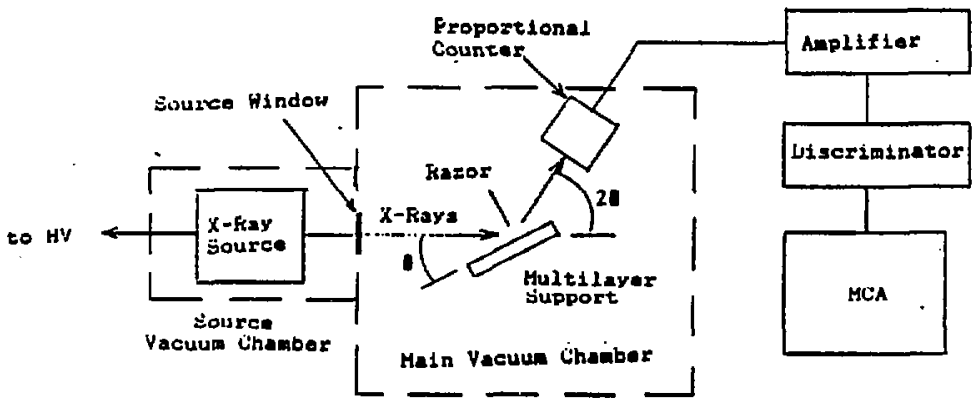

Figure 21. A block diagram of the $x$-ray spectrograph used in this report. The source vacnum is isolzted from the main vacuum by the source pindow. Not drawn is the goniometer upon which the multilayer and proportional counter are mounted.

flection data. X-rays are produced by the Hente tube $x$-ray source This radiation then pastes through a window which serves to isolate the source vacuum from the generally poorer vacuum of the main vacuum chamber. The window also serves to collimate the beam so that we have a uniform beam spot. The collimated $x$-rays then scatter off of the multilayer, passing under the edge of a razor blade which is lowered close to the multilayer 
surface. The scattered reys are then detected by a proportional counter. The counter and multilayer are mounted on a goniometer in such a way that when the multilayer is rotated through an angle $\theta$, the proportional counter will be rotated through an angle of $2 \theta$.

We proceed in our discussion by detailing further various important components of the $x$-ray spectrograph. We begin by discussing the $x$-ray source, discuss the detection apparatus, then consider how to generate a reflection profile and how to interpret the measurements.

\section{The Henke Soft X-ray Source}

Figure 22 shows a cross section of a Henke $x$-ray tube. ${ }^{12}$ To generate $x$-rays, we pass

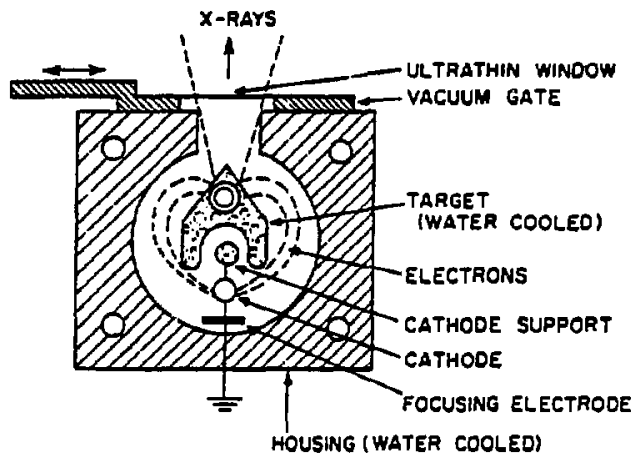

Figure 22. The Henke $x$-ray tube. Typical paths which the thermionic electrons follow from the cathode to the anode are drawn as dashed lines.

a current through the helical tungsten cathode, which then undergoes Ohmic heating. 
At a high enough temperature, electrons will bave enough kinetic energy to escape from the cathode. This process is known a thermionic emission. The anode is maintained at 2 positive potential (1kV-10kV) so the emitted electson will be sccelerated towerds it, travelling along the paths indicated in Fig. 22. The crounded focuring atrip behidd the cathode helps to constrain the electrons to the paths shown. The electrons strike the anode in two large focal spots $\left(\sim 1 \mathrm{~cm}^{2}\right)$ and excite the atoms they strike, causing the atoms to emit their characteristic lise spectrum. Thin radiation then passes out of the tube hous. ing through a thin window which mey be chowen for its x-ray transmission properties to filter out underired wavelengths. The anode and tube housing are water-cooled to prevent melting under electron bombardment. The anode is interebangeable, so we can obtain radiation at many characteristic wavelengths by chocaing an anode made of the appropriate element. If we wish to obtain characteristic madiation for a non-conductor, simply coating a copper anode with a thin layer of the non-conductor is effective. ${ }^{22}$

Several steps are taken to prevent contamination which will lead to undesired spectral lines being pieduced. First, before an anode is mounted in the $x$-ray tube, it is cleaned and its surface hested to degas it. While it is operating, the x-ray tube is constantly pumped to maintain a good vacuum ( 10-' Torn) nearly free of contaminants. Also, the position of the cathode behind the anode prevents the sublimation of tungsten upon the anode's active surfaces. We can put additional filters in the path of the x-rass outside the tube window to seduce the intensity of any contaminating lines which might be present. The filtration we use for ous various spectral lines is given in Table I.

Even without contamination, we do not only see the desired spectral lines emitted from the source - we also see a continuum background. This continuum arises from the deceleration experieneed by the electrons emilted from the cathode when they impact upon the anode. An electron which strikes the anode does not give up all of its binetic energ: all at once, but rather in a series of collisions. If an electron loses an anount of energy $\Delta E$ 


\begin{tabular}{|c|c|c|c|c||}
\hline line & E(eV) & source & PC & PC \\
filter & filter & wiodor \\
\hline B-K & 183.4 & Formrar & Mo-C-Formvar & Formvar \\
C-K & 277.0 & Kimfol & - & Kimfol \\
Cu-K & 929.7 & Cu-Formvar & Cu-Formvar & Formrar \\
Al-K & 1486.7 & Al-Kimfol & Al & Al-Kimfol \\
\hline
\end{tabular}

Table I Fiitering for various $x-$ ray wavelengths

in a collision, it will emit a photon (Bremastrablung radiation) with waveleagth hc/ $\Delta E$. The value of $\Delta E$ can range from zero ap to the full linetic energy of the electron. The zet effect of a large aumber of electrons exch undergoing a series of such collisions is to build tp an x-tay continum.

An approximate expression for the intensity of this continum as a function of wavelength has been found to be:13

$$
I(\lambda)=C i z\left(\frac{\lambda}{\alpha_{\min }}-1\right) \frac{1}{\alpha^{2}}, \lambda<\lambda_{\min }
$$

Here $Z$ is the atomic number of the anode element, $i$ is the curent across the $x$-ray tube, and $C$ is a constaxs. The esrre bas 2 bigh exesg" cuteff $\lambda_{\text {min }}$ above which the conti $\lambda$ intensity is zero. This wavelength coresponds to the light ezitted by those electzors which give up all of their linetic ene:gr in one collision. An elect:on winici ctosses a potential difierence $\Delta V$ gaizs binetic exerz: equal to $e(\Delta V)$. Thus $\lambda_{\min }=h c / c(\Delta V)$ where $\Delta V$ is the anode to cathode potential difierence, called the exciting porestial. The dependeace of the continum intersity on $Z, i$ and $\Delta V$ is shown in Fig. 23. The find the maximum of the of the contiauous spectrum by setting $a I / d \lambda=0$. From this we find that $\lambda_{l_{\text {mas }}}=1.5 \lambda_{\min }$. In other words, the energ: of the peal of the continuum expressed in e $F^{*}$ is equal to $2 / 3$ of the exciting roltage expressed in volts. When the x-ray spectrograph is 


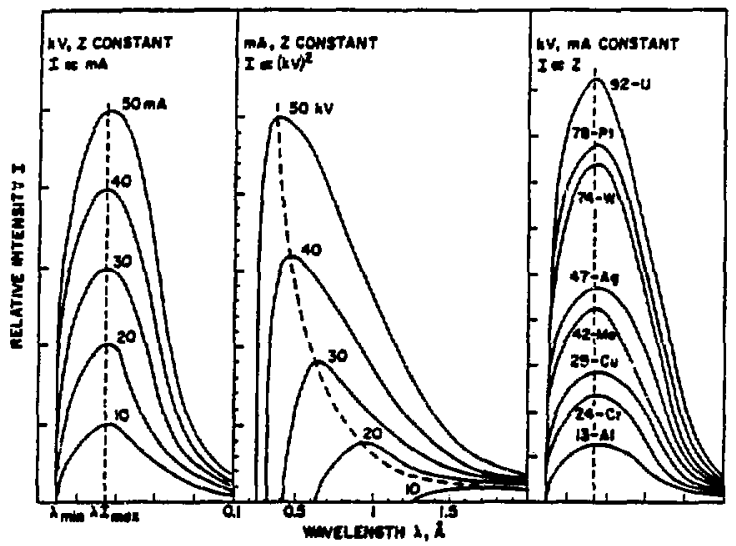

Figure 23. The effect of $x$-ray tube current, potential and atomic number of the anode on the intenuity of the continuou apectrom produced in the $x$-ray source. ${ }^{14}$

set up for measurements, we set the exciting voltage high enough so that the maximum in the continuum is at a much higher energy than the spectral line of interest. We can then electronically filter it out, a we shall discuss Inter.

\section{Detection of X-Rays}

We detect $x$-rays using a proportional counter (PC), drawn schematically in Fig. 24. The counter is filled with a detector gas, which continuously flows through the counter and out into the atmosphere at $a$ rate of about $0.1 \mathrm{ft}^{3} /$ hour. The walls of the PC act as a cathode and a thin wire which runs through the middle of the gas volume acts as an anode. X-rays enter through a thin window in the side of the PC as shown.

A proportional counter operates as follows: When a photon enters the gas volume 


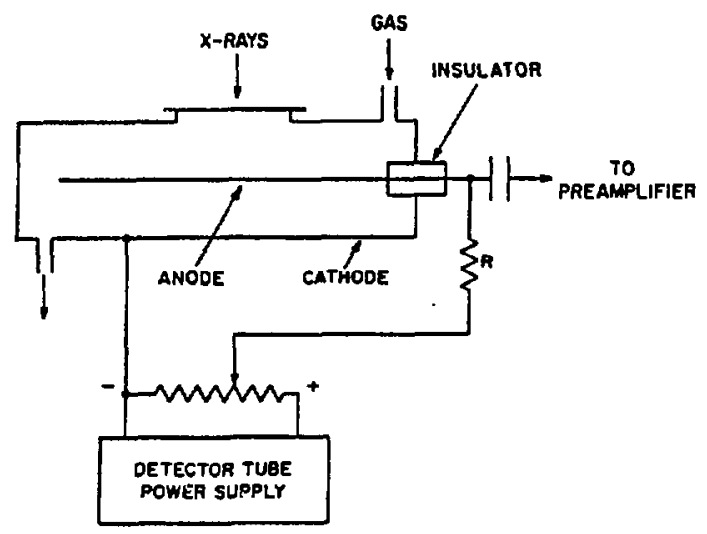

Figure 24. The structure of the proportional counter. The housing is basically cylindrical in shape and acts as a cathode. The anode wire is insulated from contact with the housing when it passes out of the proportional counter.

inside the PC, it is absorbed, creating an ion-electron pair. Virtually all of the energy of the photon is converted into the kinetic energy of the photoelectron. The photoelectron then expends its energy in collisions with neighboring gas atoms, creating more ion-electron pairs. These electrons are referred to as secondary electrons. The average number of secondary electrons produced by the photo or primary electron will be:

$$
\boldsymbol{n}_{\mathrm{t}}=E / V_{\text {eff }}
$$

where $E$ is the energy of the incident photon and $V_{\text {eff }}$ is the effective ionization potential. $V_{\text {ed }}$ will generally be a slightly higher energy than the first ionization potential of the detector gas because some gas particles may become doubly ionized and the second ionization potential is higher than the first. The number of secondary electrons actually created for a given photon will obey Poisson statistics, ${ }^{14}$ so the distribution of $n$, for a large number of photons will have a standard deviation of $\sqrt{n_{s}}$. 
All of the electrons liberated by an absorbed photon will be accelerated towards the anode. Each of these will cause a cascade of further ionizations so that a large number of electrons will eventually $(\sim 1 \mu s)$ seach the anode and form an electrical pulse. We speak of the gas gain, $G$, as the number of electrons collected by the anode, $n$, divided by the number of secondary electrons produced:

$$
G=\pi / n
$$

$G$ is typically on the order of $10^{5}$ and in independent of incident photon energy. Thus the number of electrons collected by the anode and therefore the size of the electrical pulse generated in the PC is proportional to the energy of the incident photon. This is why detectors of this type are celled proportional counters.

It is desirable for the absorption of $x$-rays in the detector gas to be high so that nearly all of the incident photons are counted. However, the abeorption must not be so high that all of the photons are absorbed in a thin layer just inside the counter window, for the window mag capture some of the secondary electrons produced, so proportionality would be lost. In practice, we choose a detector gas and gas pressure for each wavelength of radiation so that about ninety-five percent of the incident photons are absorbed within the counter volume. The gas types and pressures we use are given in Table II

The output from the proportional counter is a series of electrical pulses. Each pulse corresponds to the absorption of one photon and the pulse's amplitude or height is proportional to the pulse's energy. This output signal is amplifed and then is fed into a multichannel analyzer (MCA), which merely counts the number of pulses produced. We interpose an electronic discriminator between the amplifier and the MCA which passes only pulses within a certain height range. In this way we can count only photons within a limited range of energ;. We set the discriminator to pass only energies near the charac. teristic line energy. The contribution of the Bremsstrahlung continuum to the measured intensity is then negligible, since we have set the source voltage so that the continuum 
pestes at an energy well away from the characteristic line energy.

\section{Measurement Of Raflectivity}

To sctually measure the reflectivity of a multilayer at a particular photon energy, we first install the proper anode, filters and so forth as given in Tables I and II. Then we mount the multilayer as shown in Fig. 21, with a mazor biade lowered to a small distance - above its surface. This is done to limit the reflection seen by the proportionsl counter to come from a small area of the ergstal, and correspondingly to represent a small range of incident angles. We begin measurement at a small negative angle ( $\theta \approx-2$ degrees) so that no intensity is seen by the proportional counter. We then periodically inerement the seattering angle by $\Delta \theta$ (typically $.005^{\circ}$ to $.02^{\circ}$ ). To maintain the proper geometry so that the PC remains at the angle of reflection for the crystal, each time we rotate the multilayer by $\Delta \theta$ we aiso rotate the $\mathrm{PC}$ around the same axis by ar angle of $2 \Delta \theta$. At each angle we measure for a period of time $\Delta t$ ( 1 to 5 seconds) the number of photons which scatter off of the multilayer and are subsequently detected by the PC. In this way we build up an intensity versus incident angle curve. We continue this process until all the desired Bragg peaks have been detected. -

The number of photons seen by the PC at a given angle is not only dependent upon the reffectivity characteristies of the ergstal, but also upon the intensity of x-rays incident upon the crystal. Simply stated, if we double the incident intensity, we double the number of photons detected. Uinfortunately, due to the geometry of our spectrograph, the incident intensity varies with the angle of incidence. We now examine this angular dependence in detail.

At the start of a reflectivity run, the multilayer is positioned so that $\theta$ has a negative value, as drawn in Fig. 25a. The multilayer is sputtered onto a thick substrate, so no 


\begin{tabular}{|c|c|c|c|}
\hline line & $E(e V)$ & detector & $\begin{array}{c}\text { preseure } \\
\text { (Torr) }\end{array}$ \\
\hline B-K & 183.4 & Propane & 100 \\
C-K & 277.0 & Propane & 200 \\
Cu-K & 929.7 & Propane & 200 \\
Al-K & 1486.7 & P10 & 760 \\
\hline
\end{tabular}

Table II PC Gas and Gas Prunres for vrious x-ray wavelengths

transmission of x-rays through the bottom of the multilager is possible. The intensity detected by the $P C$ is zero. As we increment $\theta$, erentually x-rays will be able to pass through the slit between the multilayger and the razor, as damo in Fig. 25b. This begins at an angle determined by:

$$
\tan \theta=-s / a
$$

where $s$ is the perpendicular silt width and $a$ is ore-half of the length of the multilayer as showr. The ratio of $s / a$ is typically about $1 / 50$ so we may write:

$$
\theta=-s / a
$$

For 20 angle in the range of $-9 / 0<\theta<0$, the intensity will increase lineas!y mith $\theta$ :

$$
I=c(s \div a \theta)
$$

where $c$ is a proportionalizy constant.

Figure 25c show's the oriestation of the crystal when $\theta=0$. For the in:essity at this point we write $I(0)=e s$. When becorces positive we begin to have reffection of of the suface of the multilayer. At grazing incidence, the multilayer surace nill act just libe a mirror and will totally reflect soft x-rays. However, the reffected intersity may not be equal to the incident iatensity even as $\theta$ approaches zero because the soughness of the surface 


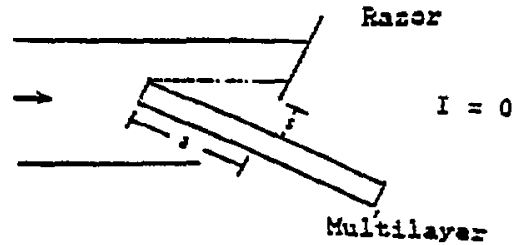

$0<-\frac{8}{4}$

A

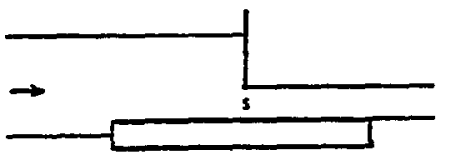

$I=\mathrm{es}$

$\theta=0$

C

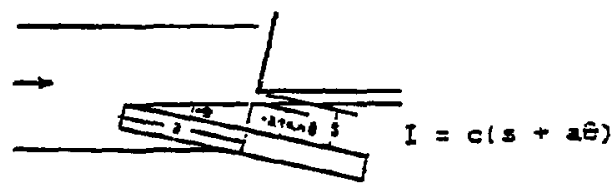

$-\frac{3}{1}<0<0$

B

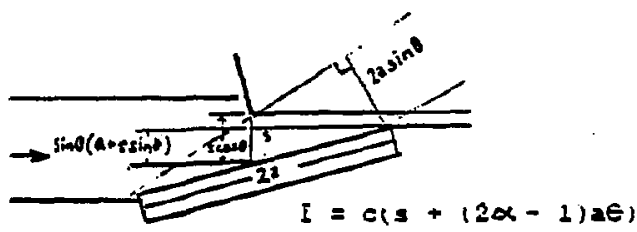

$0<0<3$

D

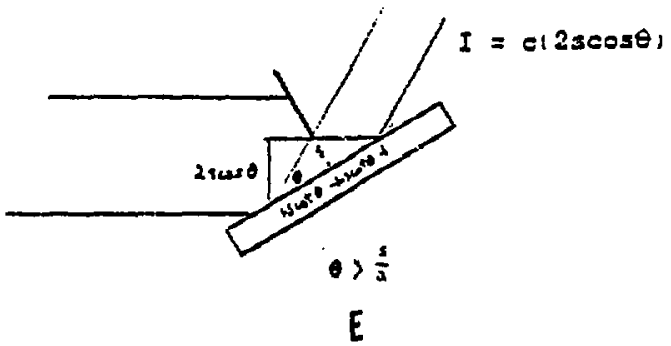

Figure 25. The geometzy for determination of the effective incident intensity for different angles of incidencen. 
will result in some loss. The intensity detected by the PC will be the sum of contributions from the intensity which passes directly through the slit and that which is reflected off of the multilayer. from the geometry drawn in Fig. 25d, this equals:

$$
I=c[s \cos \theta-(a+s \sin \theta) \sin \theta+\alpha(2 a \sin \theta)]
$$

where $\alpha$ gives the graxing incidence reflectivity of the crystal. For the angles of consideration $\left(0<\theta<\frac{4}{a}\right)$, we may write:

$$
I=c(s+(2 \alpha-1) a \theta)
$$

Again the intensity increases linearly with $\theta$, but now with a different slope. Thus we find an inflection point at $\theta=0$, as depicted in Fig. 26 for various values of $\alpha$. Typically $\alpha$ is nearly equal to one, so the change in slope may not be great.

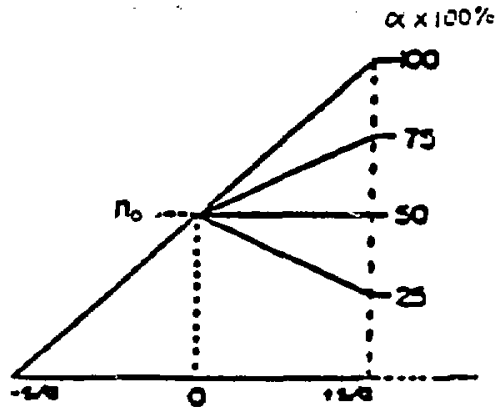

Figure 26. The $x$-ray intensity seen opponite the multilayer slit for the range $\frac{z}{a}<\theta<\frac{2}{a}$. When the grazing incidence reflectivity of the multilayer, $\alpha$, is less than one, we see an inflecition point at $\theta=0$, as shown.

At angles greater than $\theta=\frac{4}{a}$, all of the $x$-rays detected by the PC are reflected by 
the multilager, as shown in Fig. 25e. The incident intensity bere is:

$$
I=c(2 s \cos \theta)
$$

This is the region in which the Bragg peaks oceir. If we count $n$, photons at the zero angle position (determined by the location of the inflection point), then from a perfectly reflecting crgstal we expect:

$$
n=n_{\bullet} \frac{I(\theta)}{I(0)}=2 n_{0} \cos \theta
$$

photons to be counted at an angle $\theta$. We determine the crystal's real reflectivity at a given angle by dividing the actual number of photons measured, $n_{n}$, by the value of $n$ given above. For example, the measured peak reflectivity for the first Bragg order is given by:

$$
P_{1 x}=\frac{n_{x}\left(\theta_{1}\right)}{2 n_{0} \cos \theta_{1}}
$$

where $\theta_{3}$ is the first order Brags angle.

The value we are mont interested in for each of the Bragg orders is the integrated reflectivity, $R$. We use this value instead of the-peal reflectivity, $P$, for two reasons. First, each of the Bragg peaks will undergo instrumental broadening as well as broadening due to the energy width of the source line. As the curve is brosdened, the peak ralue is correspondingly decreaced. Fowever, $R$ remsins constant, so we can avoid estimating the amount of broadening that oscurs for a given arsagement of the spectrograph by merely using $R$. Second, the statistical error in $R$ is smaller than that in $P$. Both $R$ and $P$ will obey Poisson statistics, so the statistical error for each of these is:

$$
\operatorname{err}(P)=\frac{1}{\sqrt{n P}} \times 100 \% \operatorname{err}(R)=\frac{1}{\sqrt{n \pi}} \times 100 \%
$$

where $n_{R}$ is the number of counts for $R$ and $n_{P}$ is the number of counts for $P$. The value of $n_{R}$ will generally be many times that of $n_{P}$, so the error in $R$ will be much smaller than the error in $P$. As a typieal example, we may have $n_{R}=100000$ and $n_{P}=1000$. The statistical errors in $R$ and $P$ are then approsimately 0.3 percent and three percent, 
respectively.

We have not yet stated over what range $R$ will be integrated for each order. We wish to retain as much of the Bragg peak as possible and so may wish to integrate over a wide range of angles about the peak. Howrever, the number of counts in the tails of the Bragg peak will be low, so if there is a small amount of background intensity in our measurements, the aree in this background may be greater then that of the tail, and we are increasing the error in $R$. In addition, the MDP expreasion is only valid near the Brags peak, where $\xi$ is small. In this report we define $R$ over a range of \pm 2 FWHM $(\omega)$ around the position of peak intensity, $\theta_{P}$, as shown in Fig. 27. This retnins about nizety percent of the total area of the peak and minimizes the problems ascociated with the tails of the curve.

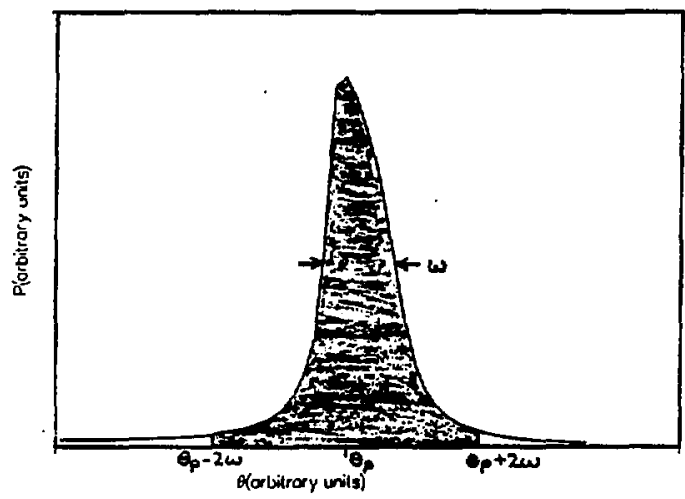

Figure 27. The integrated reflectivity is defined over a range of $\pm 2 \omega$. It is represented here for 2 typical Bragg peak at the shaded area.

As mentioned earlier, a multilayer crystal will reflect $x$-rays from its surface at small angles. This is known as total reflection, and is calculated from the usual Fresnel equations 
for the reflection of electromagnetic waves at the interface between two materials. For the interface between the multilayer and the sursounding vacuum we write: ?

$$
\begin{aligned}
& \left(\frac{E_{R}}{E_{I}}\right)_{r}=\frac{2 \sin \theta}{\sin \theta+\sqrt{n_{I}^{2}-\cos ^{2} \theta}} \\
& \left(\frac{E_{R}}{E_{l}}\right)_{\cdot}=\frac{\sin \theta-\sqrt{n_{I}^{2}-\cos ^{2} \theta}}{\sin \theta+\sqrt{n_{I}^{2}-\cos ^{2} \theta}}
\end{aligned}
$$

where $n_{x}$ is the complex index of refraction of the surface material, which may be written $1-\delta-i \beta$, where $\delta$ and $\beta$ are amall compared to one. $\left(E_{R} / E_{I}\right)_{r}$ and $\left(E_{R} / E I\right)_{\text {, are the }}$ ration of the reflected wave amplitude to the incident wave amplitude for light polarized parallel and perpendicular to the plane of seattering, respectively. For unpolarized light, we average the contributions of the $\pi$ and $\sigma$ polarizations. If a Bragg peak occurs at a small enough angle, it may overlap with the tail of the total reflection curve. Figure 28 demonstrates this for a real multilayer. Unfortuantely the MIDP theory cannot be used to model the total reflection in the vicinity of a Brags penk. We treat this problem as follow: If a Bragg peak; is outside of the total reflection region, as shown in Fig. 29a, we hare no problem and calculate $R$ normally. If the total reflection tail is small with respect to the Bragg peat; we may approsimate the tail as a linear function and subtract its area out from the integrated reflectivity as in Fig. 20b. If the total reflection tail is large in the vicinity of the Bragg peak, as in Fig. 29c, we cannot easily deconvolve its area with that of the peal. In this case we canoot use the order in the fitting process. Fie arbitrarily choose the cutoff point for this to be when the inisnsity at $\theta_{P}-2 \omega$ equals twenty percent of the peal intensity. 


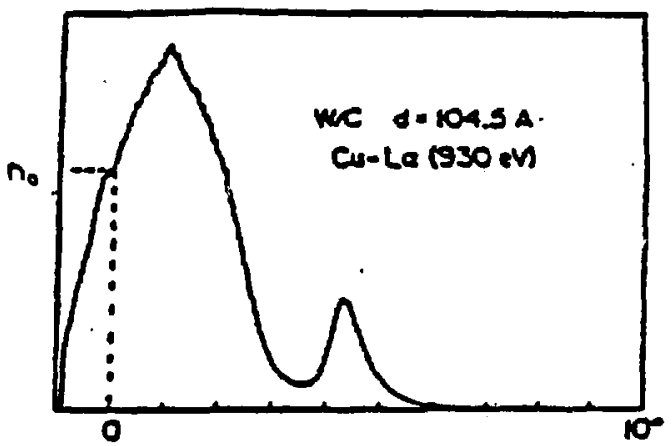

Figure 28. At small anglex, the Bragg peat may compete with the total reflection curve. Notice the inflection point at $\theta=0$.

\section{DATA AND ANAIYSIS}

As was mentioned earlies, we have been sble to fit the reflectivity characteristics of several tungaten-carbon and vanudium carbon crystals using the MDP theory and a two parameter model of the usit cell. However, we found two parameter fitting to be insufficient to madel molybdenum- silicon or vanadium-nilicon multilayers. We will firat consider the two parameter fits obtained for these multilayers then compare them to our recent three parameter fits.

We attempted to flt two Mo-Si and two Si-V crytal received from Energy Conversion Device Corporation (ECD), a well a one Si-Mo cryatal made at the Center for X-Ray Optics (CXRO) at Lawrence Berkeley Labs. Here we have listed the element which appears as the top layer in the crystal frat (this is the $\mathrm{X}$-element in our model). The basic 


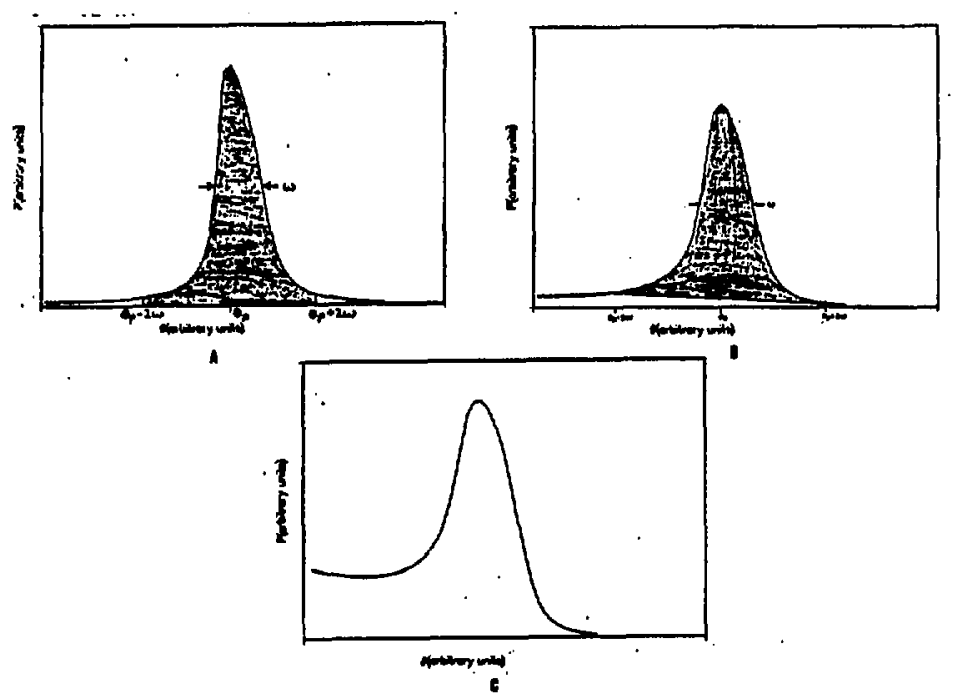

Figure 29. Varying amounts of competition between the Pisigg peak and the total reflection curve and how we define the integrated rellectivity in each case. a) no competition. b) a small total reflection backgronod is subtracted out. c) a large baciground prevents a value for $R$ from being determined.

characteristics of these crystals are given in Table III. To designate a particular crystal, we give its componition followed by its approximate $2 d$ ralue. Thus SiV145 refers to the silicon-vanadium crystal with a $2 d$ value approximately equal to $145 \mathrm{~A}$.

We measured the reflectivity profiles of these crystals to as many as four Bragg orders at three photon energies. We used the C-K $(277.0 \mathrm{eV}), \mathrm{Cu}-\mathrm{L}(929.7 \mathrm{eV})$, and Al-K (1486.7 eV) lines for the vanadium-ailicon cryotals. For the molybdenum-silicon crystals, we substituted B-K $(183.4 \mathrm{eV})$ line for C-K line because the reflectivity for this type of cryutal is low for C-K radiation.

To obtain a beat fit characterization of the uait cell, we varied the two parameters, $Y$ and $T$, until the mean relative error for all the measured orders was minimized. For $N$ measured orders, the function we minimized is:

$$
\operatorname{err}=\left[\frac{1}{N} \sum_{i=1}^{N} \frac{R_{i(\text { heorg })}-R_{i(e x p))}}{R_{i(e s p)}}\right]^{\frac{1}{2}}
$$




\begin{tabular}{||c|c|c|c|c||}
\hline $\begin{array}{c}\text { cryatel } \\
\text { dexignation }\end{array}$ & source & $\begin{array}{c}\text { composition } \\
(\mathrm{X}-\mathrm{Y})\end{array}$ & $2 d$ & $N$ \\
\hline MoSi166 & ECD & $\mathrm{MO}-\mathrm{Si}$ & 166.0 & 95 \\
MoSi198 & ECD & $\mathrm{Mo-Si}$ & 197.8 & 60 \\
SiMo197 & CXRO & Si-Mo & 196.6 & 30 \\
SiV145 & ECD & Si-V & 145.2 & 60 \\
SiV210 & ECD & Si-V & 209.7 & 60 \\
\hline
\end{tabular}

Table III. Crystal characteristics for the multilayess studied in this report.

This fitting was performed antomatically by a FORTRAN code which uses a standard minimization routine on a VAX computer. The parameters we found for each crystal are given in Table $\Gamma$. Table $V$ lists the meanured integrated reflectivity values and the

\begin{tabular}{|c|c|c|c|}
\hline $\begin{array}{c}\text { crystal } \\
\text { decipmation }\end{array}$ & $(\boldsymbol{X})$ & $\mathrm{T}$ & err \\
\hline MoSi166 & 14.0 & 19.6 & .318 \\
MoSi198 & 24.8 & 19.0 & .488 \\
SiM0197 & 18.2 & 11.9 & .424 \\
SiV145 & 15.9 & 16.0 & .492 \\
SiV210 & 39.7 & 15.4 & .615 \\
\hline
\end{tabular}

Table IV. Resulting characterization from two parameter fitting.

theoretical values based on the parameters of Table IV. These fits are unacceptably poor for fourth order Bragg reflection, where the theoretical reflectivity is often off by a factor of ten or more from the measured values.

In ordex to more accurately characterize the molybdenum-silicon and vanadiuza-silicon multilayers, we turn to the three parameter model outlined in this report. We perform 


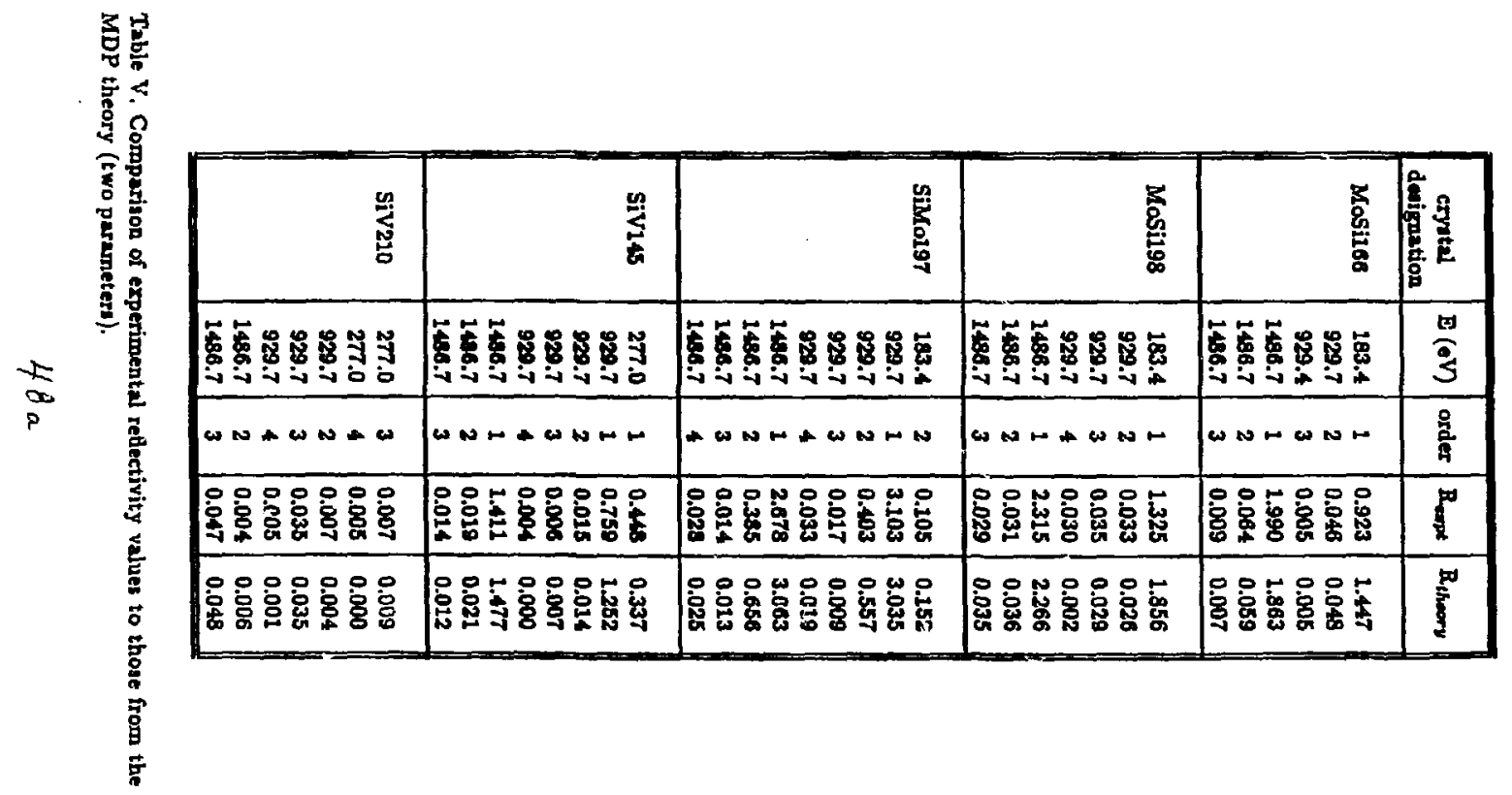


the fitting procedure just as before, now vaging the ralues of $Y, T_{1}$ and $I_{2}$ to bert match the model to the experimental integrated reflectivity values. These parameterizations are gires in Table VI and a comparison betwean the expecimental and theoretical refectivities is given in Table VII The fit are subetantially iniporved for all of the corstals, eroecially

\begin{tabular}{|c|c|c|c|c|}
\hline $\begin{array}{c}\text { erystal } \\
\text { dosignetion }\end{array}$ & $\begin{array}{c}Y \\
(\AA)\end{array}$ & $T_{L}$ & $T_{2}$ & 2. \\
\hline MoSi166 & 18.9 & 18.8 & 38.2 & .120 \\
Mo5i198 & 25.7 & 10.4 & 32.5 & .318 \\
SiM0197 & 19.9 & 20.6 & 7.5 & .207 \\
SiV145 & 20.9 & 25.6 & 8.5 & .227 \\
SiV210 & 37.2 & 23.8 & 7.2 & .358 \\
\hline
\end{tabular}

Table VL Resulting characterization from three parameter fitting.

for the fouth oxder reflectivitie. The grater inprovenent in the higher oxdess is to be apected, for theg are more sentitiva to rasiations is the eystal parmeterization (see, for enmple, Fig. 20).

The parametes we are mont intereated in ase the tranition layes thicknesses, $T_{1}$ and $\boldsymbol{T}_{2}$, for we epect that thase thicleieses should ba approximately the same for different multilay ers of the same trpe. In fact, we see ereflent agreement in these values for the two ranadium- silicon muitilizers. For the molybdenum-silicon muitilayers the agreenent is not as good-the crogtal sputtered at CXRO has much smaller interince thichosses than those sputtered at ECD. This may be due to difierences in the sputtering conditions at the two locations. The two ECD erstals, MOSiS3 and MIOSig9, still show a moderate diserepancy between them 


\begin{tabular}{|c|c|c|c|c|}
\hline $\begin{array}{c}\text { cryatal } \\
\text { decigantion }\end{array}$ & $\mathbf{E}(\mathbf{e V})$ & order & Rرسما & $\mathbf{R}_{\text {thery }}$ \\
\hline MoSil66 & $\begin{array}{r}183.4 \\
929.7 \\
929.4 \\
1486.7 \\
1496.7 \\
1486.7 \\
\end{array}$ & $\begin{array}{l}1 \\
2 \\
3 \\
1 \\
2 \\
3 \\
\end{array}$ & $\begin{array}{l}0.923 \\
0.046 \\
0.005 \\
1.990 \\
0.064 \\
0.009 \\
\end{array}$ & $\begin{array}{l}0.941 \\
0.047 \\
0.005 \\
1.868 \\
0.059 \\
0.007 \\
\end{array}$ \\
\hline MoSi198 & $\begin{array}{r}183.4 \\
929.7 \\
929.7 \\
929.7 \\
1486.7 \\
1486.7 \\
1486.7 \\
\end{array}$ & $\begin{array}{l}1 \\
2 \\
3 \\
4 \\
1 \\
2 \\
3\end{array}$ & $\begin{array}{l}1.325 \\
9.033 \\
0.035 \\
0.030 \\
2.315 \\
0.031 \\
0.029 \\
\end{array}$ & $\begin{array}{l}1.695 \\
0.029 \\
0.027 \\
0.013 \\
2.550 \\
0.036 \\
0.032\end{array}$ \\
\hline SiMo197 & $\begin{array}{r}183.4 \\
929.7 \\
929.7 \\
929.7 \\
929.7 \\
1486.7 \\
1496.7 \\
1486.7 \\
1486.7 \\
\end{array}$ & $\begin{array}{l}2 \\
1 \\
2 \\
3 \\
4 \\
1 \\
2 \\
3 \\
4\end{array}$ & $\begin{array}{l}0.105 \\
3.103 \\
0.403 \\
0.017 \\
0.033 \\
2.678 \\
0.385 \\
0.014 \\
0.028 \\
\end{array}$ & $\begin{array}{l}0.107 \\
3.233 \\
0.350 \\
0.012 \\
0.023 \\
3.254 \\
0.421 \\
0.017 \\
0.031\end{array}$ \\
\hline SiV145 & $\begin{array}{r}277.0 \\
929.7 \\
929.7 \\
929.7 \\
929.7 \\
1496.7 \\
1496.7 \\
1486.7 \\
\end{array}$ & $\begin{array}{l}1 \\
1 \\
2 \\
3 \\
4 \\
1 \\
2 \\
3\end{array}$ & $\begin{array}{l}0.448 \\
0.759 \\
0.015 \\
0.006 \\
0.004 \\
1.411 \\
0.019 \\
0.014\end{array}$ & $\begin{array}{l}0.336 \\
1.082 \\
0.014 \\
0.007 \\
0.004 \\
1.314 \\
0.020 \\
0.012 \\
\end{array}$ \\
\hline Siv210 & $\begin{array}{r}277.0 \\
277.0 \\
929.7 \\
929.7 \\
929.7 \\
1486.7 \\
1486.7\end{array}$ & $\begin{array}{l}3 \\
4 \\
2 \\
3 \\
4 \\
2 \\
3\end{array}$ & $\begin{array}{l}0.007 \\
0.005 \\
0.007 \\
0.035 \\
0.005 \\
0.004 \\
0.047\end{array}$ & $\begin{array}{l}0.008 \\
0.002 \\
0.004 \\
0.034 \\
0.005 \\
0.006 \\
0.047\end{array}$ \\
\hline
\end{tabular}

Table Vll Comparison of experimental reflectivity values to those from the MDP theory (three purameters).

$$
49 a
$$


Unfortunately we do not know whether the sputtering conditions for these two multilayers were the same. Petford-Long et. al. report the trannition region thicknessen of two molybdenum-ilicon crystals to be $17 \AA \pm 3$ Afor Mo oputtered onto Si interfaces and 10 $A \pm 3$ Afor Si on Mo interfaces. ${ }^{15}$ These multilayers were sputtered at Lawrence Livermore National Laboratory, and were measured using transition electron microscopy (TEM) techniques. These interface thicknesees agree reasonably well with our values for the multilayer manufactured at CXRO.

All of the multilayers we measured show values of $T_{1}$ and $T_{2}$ which differ by a factor of two or more. In all cases, the transition region produced when the heavy element is sputtered onto the light element is much thicker than the transition region produced by the reverse process. To understand this, let us consider the sputtering of a molybdenumsilicon crystal. Because a Mo atom is much more massive than a $\mathrm{Si}$ atom, Mo is sputtered with an average kinetic energy correspondingly greater than that of Si. ${ }^{16}$ Thus Mo atoms impinging upon a silicon surface may penetrate further than $\mathrm{Si}$ atoms impinging upon a molybdenum surface. In addition, sputtered molybdenum films are for the most part crystalline, whereas silicon films are amorphous. Thus atoms can penetrate the rather open silicon structure more easily than the densely packed crystalline structure of the molybdenum surface. ${ }^{15}$ Also, the amorphous $\mathrm{Si}$ will have a rougher surface than the crystalline Mo, so even without surface penetration, tine Mo on Si interface region will be thicker than the Si on Mo surface. These considerations should also apply to vanadium-silicon interfaces.

It would seem that these considerations should apply also to such multilayer types as tungsten-carbon and vanadium-carbon, and so we might expect to see a large difference in the interface thicknesses of these multilayers an well. However, this was not the case-we were able to use the two parameter model to fit these types. This can be explained if we consider the important differences between carbon and silicon. Both of these elements form amorphous layers when sputtered, but carbon forms a more compact structure, and 
the energy necessary to liberate a carbon atom from this structure is several times the energy necessary to liberate a silicon atom from its less dense structure ${ }^{17}$. Thus we expect that atoms will not be able to penetrate a carbon surface as deeply as a silicon surface. Also, because carbon is much less massive than silicon, it will not penetrate surfaces as deeply. These considerations imply that the transition regions of multilayers with carbon as the light element will be thinner than those of multilayers with silicon as the light element. This is in fact what we observe-the average transition layer thickness for the two vanadium- silicon multilayers in this report is about $16 \AA$; for vanadium carbon multilayers we found an average value about half of this ${ }^{5}$. Multilayers such as vanadium-carbon may in fact have interfaces of appreciably different thicknesses, but our model is not sensitive to this difference when the thicknesses are small (see Fig. 20).

Although we can obtain reasonably good characterizations with our present model, it has several limitations which we now discuss. First, our model is based upon a perfectly periodic layer system. Effect! such as layer to layer variations of the d-spacing and lateral variations in the crystal characteristics are not accounted for. Also, we have used the bulk densities of the two elements in our calculations, whereas the actual densities of sputtered filma may be somewhat lower. For most multilayera, however, we expect that the error introduced to the reflectivity values by these effects will be small, as films generated using current R-F sputtering techniques are usually uniform and have layer densities which approsch bulk values. ${ }^{2}$

The tabulated values of the atomic scattesing factors for a given element may not be very reliable near its absorption edges. For this reason, we cannot use measurements made at these energies for characterizing multilayers. For instance, we would not measure the reflectivity of a molybdenum-silicon crystal using Si-L radiation (91.5 eV).

Another limitation of our model is that it is only valid when the absorption per layer is small. This means that we cannot use the model for crystals with large d-spacing values 
and at long wavelengths. As an example, we cannot use measurements of the SiV210 crystal made at the B-K line (183.4 eV) because the absorption per layer, $\sigma$, has a magnitude of about 0.4 at the first Bragg order. Our calculations assume that $\sigma$ is small compared to one, which does not hold in this case. Finally, we have modelled the interface structure as a linear function. This is a reasonable first approximation, but it has been suggested that a more precise model would have a Gausoian form, such as is drawn in Fig. $30^{18}$. We might expect such a model to better fit the higher orders, as it is these orders which are most sensitive to changes in the parameterization of the unit cell.

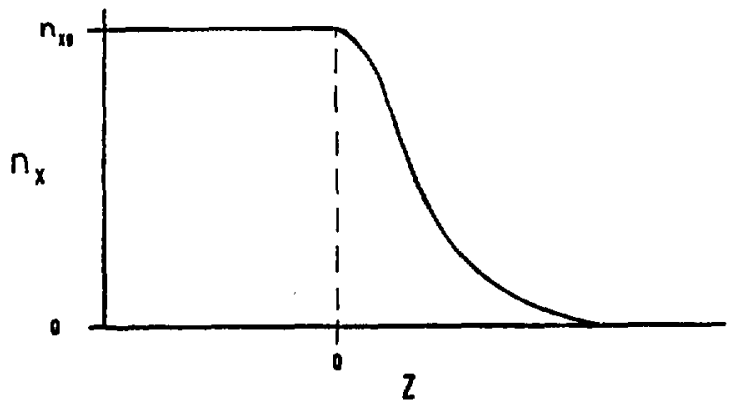

Figure.30. The Gaussian interface structure suggested by Chauvineau et. al. It appears analytically as: $n_{x}=n_{x o} e x p\left(-z^{2} / 2 \sigma\right)$ for $z>0 . \sigma$ is a measure of the width of the transition region. 


\section{CONCLUSIONS}

The ability to quickly and accurately characterize arbitrary multilayers is very valuable for not only can we use the characterizations to predict the reflectivity of a multilayer for any soft I-ray wavelength, we also can generalize the results to apply to other multilayers of the same type. In addition, we can use the characterizetions as a means of evaluating various sputtering environments and refining sputtering techniques to obtain better multilayers.

In this report we have obtained improved characterizations for sample molybdenumsilicon and vanadium-silicon multilayers. However, we only examined five crystals overall, so the conclusions that we could draw about the structure of general multilayers is limited. Research involving many multilayers manufactured under the same sputtering conditions is clearly in order. In order to best understand multilayer siructures it may be necessary to further refine our model, eg., adopting a Gaussian form for the interface regions. With such improvements we can expect even better agreement with experimental values and continued concurrence with other characterization teciniques. 


\section{ACKNOWLEDGEMENTS}

The authors gratefully acknorvledge the invaluable assistance of A. R. Hashima, J. A. Kerner and R. C. C. Perera in preparation of this report. This worl was supported by the U.S. Department of Energy under contract No. DE-AC03-76SF00098. 


\section{REFERENCES}

1. J.B. Kortright, P. Plag, R.C.C. Perera, P.L. Cowan, D.W. Lindle, and B. IKariin, Nucl. Inst. and Methods (in press).

2. T.W. Barbee, Ir., Proc. of SPIE 863, 2 (1985).

3. R.V. Sturat, Vacuam Technology, This Films, and Sputtering, Academic Press, Niew Yorle (1983).

4. M.P. Bruiju, P. Chairaborty, E. ne Esen, J. Verhoeven and M.J. van der Wiel, Proc. of SPIE 563, 182 (1985).

5. B.I. Henke, J. Kerner, and A.L. Oren, (to be published).

6. A.H. Compton and S.K. Allison, X-R2ys in Theory and Experiment, 2nd ed., Van Norstrand, New York (1935).

7. R.W. James, The Optical Principles of Diffaction of X-Rays, Cornell University Press, Ithace, New York (1965).

8. B.L. Eeake, J.Y. Uejio, H.T. Yamads, and R.E. Taclaberry, Opticel Engineering, 25(3), 937 (1986)

9. J.D. Jackson, Classical Electrodynamics. J. Wiley and Sons, Niew York (1962).

10. B.L. Heake, P. Lee, T.J. Tanala, R.L. Shimabuluro and B.K. Fujiliawa, dtomic and Data Tables, 27 (1982).

11. O.P. Rugsti, J. Opt. Soc. Am., 55,630 (1963).

12. B.L. Herke, Advances in X-Ray Aral., 5, 2S5 (1962).

13. E.A. Iiramers, Phil. Mag. 46, 836 (1923). 
14. E.P. Bertin, Prizciplea and Practice of X-Ray Spectrometric Analysis, 2nd ed., Pleaum Press, New York (1975).

15. A.K. Petford Long, M.B. Stearn, C-E. Chang, S.R. Nutt, D.G. Stearns, and A.MI. Hawryluk, J. Appl. Phys., 61(4), 1422 (1987).

16. D.G. Stearas, N.M. Ceglio, A.M. Fawryluk, M.B. Stearns, A.K. Petford-Long, C-Z. Chang, K. Danzmand, M. Kuhne, P. Muller, and B. Wende, Proc. of SPIE, 688, 91 (1986).

17. V. Bodart, Ph.D. Thesis, Universite de Paris - Sud, (1987).

I8. J.P. Chaurinean, J. Corno, D. Decanini, L. Nevot, B. Pardo, Proc. of SPIE, 563, 245 (1985). 